\title{
THE CARNEGIE-IRVINE GALAXY SURVEY. IV. A METHOD TO DETERMINE THE AVERAGE MASS RATIO OF MERGERS THAT BUILT MASSIVE ELLIPTICAL GALAXIES
}

\author{
SONG HuANG (黄崧) ${ }^{1}$ LUIS C. $\mathrm{HO}^{2,3}$, CHIEN Y. PENG ${ }^{4}$, ZHAO-YU Li (李兆聿 $)^{5}$, AND AARON J. BARTH ${ }^{6}$ \\ The Astrophysical Journal, Accepted
}

\begin{abstract}
Many recent observations and numerical simulations suggest that nearby massive, early-type galaxies were formed through a "two-phase" process. In the proposed second phase, the extended stellar envelope was accumulated through many dry mergers. However, details of the past merger history of present-day ellipticals, such as the typical merger mass ratio, are difficult to constrain observationally. Within the context and assumptions of the two-phase formation scenario, we propose a straightforward method, using photometric data alone, to estimate the average mass ratio of mergers that contributed to the build-up of massive elliptical galaxies. We study a sample of nearby massive elliptical galaxies selected from the Carnegie-Irvine Galaxy Survey, using two-dimensional analysis to decompose their light distribution into an inner, denser component plus an extended, outer envelope, each having a different optical color. The combination of these two substructures accurately recovers the negative color gradient exhibited by the galaxy as whole. The color difference between the two components $(\langle\Delta(B-V)\rangle \simeq 0.10$ mag; $\langle\Delta(B-R)\rangle \simeq 0.14 \mathrm{mag}$ ), based on the slope of the $M_{*}$-color relation for nearby early-type galaxies, can be translated into an estimate of the average mass ratio of the mergers. The rough estimate, $1: 5$ to $1: 10$, is consistent with the expectation of the two-phase formation scenario, suggesting that minor mergers were largely responsible for building up to the outer stellar envelope of present-day massive ellipticals. With the help of accurate photometry, large sample size, and more choices of colors promised by ongoing and future surveys, the approach proposed here can reveal more insights into the growth of massive galaxies during the last few Gyr.
\end{abstract}

Subject headings: galaxies: elliptical and lenticular, $\mathrm{cD}$ - galaxies: formation — galaxies: photometry — galaxies: structure - galaxies: surveys

\section{INTRODUCTION}

\subsection{Two-phase Formation Scenario for Massive Galaxies}

Recent observations established that massive, quiescent early-type galaxies (ETGs) at high redshift are surprisingly more compact (Daddi et al. 2005; Trujillo et al. 2006) than their descendants today. Since $z=2$, these systems on average doubled in stellar mass and increased in size by a factor of 3-5 (Szomoru et al. 2012; van der Wel et al. 2014). A "twophase" scenario (Oser et al. 2010) emerged as a plausible explanation for this dramatic evolution: intense dissipative processes rapidly built up an initially compact progenitor; then, after star formation was quenched, gas-poor ("dry"), minor mergers 7 dominated the second phase of protracted evolution. The inside-out growth of massive galaxies predicted by this picture has gained much recent observational support (e.g., Patel et al. 2013; Huang et al. 2013b). The key feature of this scenario is the dominant role of minor, mostly dry, mergers in the buildup of the extended envelope, the feature most responsible for the dramatic size growth, of massive elliptical galaxies. Many recent simulations have confirmed this and also predicted either $M_{*}$-weighted or number-weighted mass ratio for mergers during the second phase. Using re-simulations of 40 massive halos, Oser et al. (2012) concluded that "minor mergers with a mass-weighted mass ratio of 1:5" dominate the accretion events at $z<2$. Tracing the evolution of 611 massive halos in an adaptive mesh-refinement simulation, Lackner et al. (2012) also concluded that accretion onto massive halos are not dominated by major mergers, even though the fraction of the accreted component estimated by these authors is quite different from that of Oser et al. (2012). Lackner et al. also suggested that the median mass-weighted mass ratio should be smaller than 1:5. A similar conclusion was reached by Gabor \& Davé (2012): the mass growth of massive galaxies after they became quiescent was dominated by minor mergers with typical a mass ratio of $1: 5$.

Using N-body simulations of dry major and minor mergers, Hilz et al. (2012) showed that minor mergers can be very efficient in increasing the size once the dark matter halos are considered. Later, Hilz et al. (2013) demonstrated that a few mergers with mass ratios of 1:5 can explain the observed size evolution of massive ETGs. Oogi \& Habe (2013) performed "sequential minor mergers with parabolic and head-on orbits," including the dark matter halo for the satellites. Minor mergers with mass ratios between 1:10 and 1:20 are able to reproduce the observed growth in size and change of mass profile. The dry

\footnotetext{
${ }^{1}$ Kavli Institute for the Physics and Mathematics of the Universe, Todai Institutes for Advanced Study, the University of Tokyo (Kavli IPMU, WPI), Kashiwa 277-8583, Japan

${ }^{2}$ Kavli Institute for Astronomy and Astrophysics, Peking University, Beijing 100871, China

${ }^{3}$ Department of Astronomy, School of Physics, Peking University, Beijing 100871, China

${ }^{4}$ Giant Magellan Telescope Organization, 251 South Lake Avenue, Suite 300, Pasadena, CA 91101, USA

${ }^{5}$ Key Laboratory for Research in Galaxies and Cosmology, Shanghai Astronomical Observatory, Chinese Academy of Sciences, 80 Nandan Road, Shanghai 200030, China

${ }^{6}$ Department of Physics and Astronomy, 4129 Frederick Reines Hall, University of California, Irvine, CA 92697-4575, USA

${ }^{7}$ The distinction between major and minor merger in terms of mass ratio is quite arbitrary. The threshold most commonly adopted in the literature is a mass ratio of $1: 3$, which we also use here.
} 


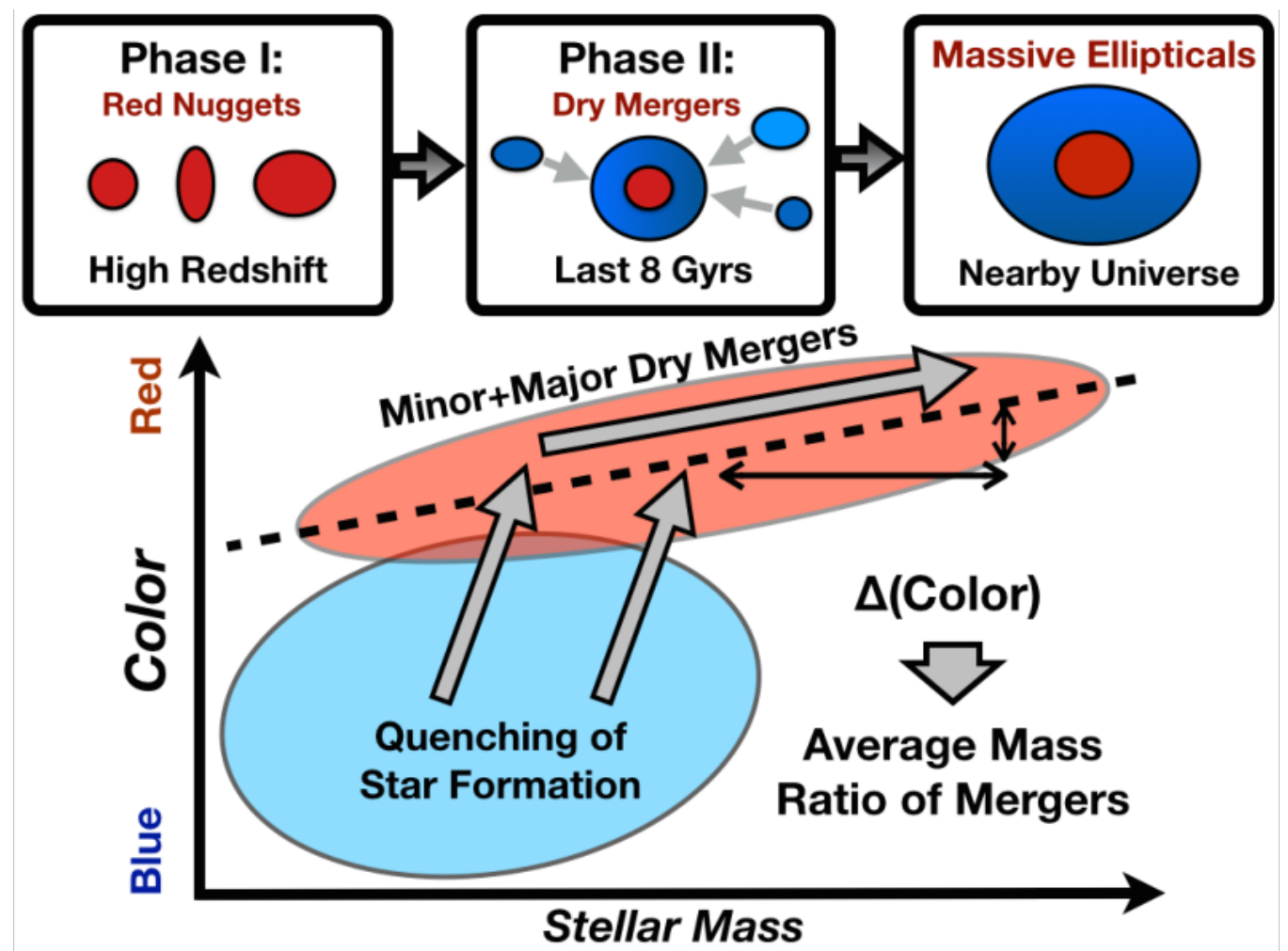

FIG. 1. - Cartoons that illustrate the basic idea of the proposed method for the estimation of average merger mass ratio. The style is adopted from Faber et al. (2007). The upper panels show the basic picture of the two-phase formation scenario of massive ellipticals. The lower panel describes a general picture for the evolution of massive galaxies on the $M_{*}$-color plane. After massive ETGs were quenched, they quickly moved to the red sequence and gradually evolved along it as they became redder due to the aging of the stellar population and more massive due to the accumulation of extended stellar envelope through many minor (dry) mergers. Since the stars of smaller systems should have lower metallicity, the outer envelope naturally has a bluer color compared to the inner region. The color difference between the inner region and the outer envelope thus reflects the average mass ratio of all mergers that contributed to the second phase of the evolution. Larger color difference means more stellar material came from smaller galaxies, making minor merger more important.

minor merger hypothesis was also tested by Bédorf \& Portegies Zwart (2013), who, again, favored minor mergers with mass ratios between 1:5 to $1: 10$.

Although various observations point to the same direction (e.g., Coccato et al. 2010, 2013; Greene et al. 2013; Lee et al. 2013), it is not clear whether enough minor mergers during the last 8-10 Gyr can account for the growth in size and mass of ETGs (e.g., Bluck et al. 2012; Newman et al. 2012; López-Sanjuan et al. 2012; Ferreras et al. 2014; Ownsworth et al. 2014). Evidence of minor mergers is plentiful in the local Universe (e.g., Tal et al. 2009; Gu et al. 2013), but they are challenging to observe at higher redshift and hard to use for quantifying the merger mass ratio. Satellite statistics provide another approach to constrain merger mass ratios. Ruiz et al. (2014) estimated the distribution of $M_{*}$ of satellites around nearby, massive galaxies in the Sloan Digital Sky Survey (SDSS) down to a mass ratio of 1:400, and concluded that the merger channel should be dominated by minor mergers with mass ratio 1:10. However, this method is quite sensitive to uncertainties in the estimations of $M_{*}$ and the mass completeness of the sample. Meanwhile, valuable fossil information embedded in the outskirts of nearby massive galaxies should also be explored to provide better observational constraints of their merging history. Arnold et al. (2011) measured the metallicity gradient of globular clusters in NGC 3115, and suggested its halo was formed through accretions of small objects with 15:1 to 20:1 mass ratio. Greene et al. $(2012,2013,2015)$ measured spatially resolved stellar population properties of $\sim 100$ nearby ellipticals out to $2.5 R_{e}$. The lower metallicity and high $\alpha$-element enhancement at large radii indicate a possible connection with smaller systems whose star formation was quenched at early time. Although globular clusters are unique tracers of formation at early phase, and spectroscopic information at large radii is very promising in light of ambitious surveys such as MaNGA (Bundy et al. 2015), these observations are very expensive to acquire for a representative sample of galaxies. Even with MaNGA, accurate stellar population properties are still hard to obtain for the faint outskirts of individual galaxies. And neither method can be applied easily to high redshifts.

Photometric colors also carry key information about the stellar population, and compared to the above-mentioned methods, are significantly easier to measure at large radii or high redshift. In this paper, we will show that the average merger history of nearby massive galaxies can be constrained using the spatial distribution of their optical colors.

\subsection{Color Gradients of Massive Elliptical Galaxies}

It is well known that massive ETGs often show a negative gradient of optical color, with colors becoming redder toward smaller radii (de Vaucouleurs 1961; Vader et al. 1988; Franx \& Illingworth 1990; Peletier et al. 1990; see Kormendy \& Djorgovski 1989 for a comprehensive review). With the help of new observations, this important feature has been characterized using much larger samples, and observed at higher redshifts (Hinkley et al. 2001; Michard 2005; Ferreras et al. 2009; La 
Barbera et al. 2010; Tal \& van Dokkum 2011). The negative gradient is usually considered to be caused by radial variation of stellar population, especially metallicity (e.g., Ogando et al. 2005; Spolaor et al. 2010; Kuntschner et al. 2010; La Barbera et al. 2010; Rawle et al. 2010). Numerous attempts have been made to explain color gradients within the formation scenario of massive galaxies. Generally speaking, intense in-situ star formation, as predicted in early monolithic collapse models, tends to leave behind a steep, negative color gradient (e.g., Eggen et al. 1962; Larson 1975; Carlberg 1984; Kawata \& Gibson 2003; but also see Pipino et al. 2010), while major merger models (e.g., White 1978) predict significant flattening of the gradient (e.g., Kobayashi 2004; Rupke et al. 2010).

Meanwhile, the two-phase formation scenario can naturally explain the negative color gradient: minor mergers tend to redistribute stars from the less massive system, which has lower stellar metallicity, to the outer envelope of the remnant (e.g., Hilz et al. 2012), and help build up a bluer stellar halo (La Barbera et al. 2013; D’Souza et al. 2014). Recently, Hirschmann et al. (2015) demonstrated that the color gradients of massive galaxies are largely due to the metallicity difference between the inner in-situ and outer accreted components (see their Figure 4). Although the negative color gradient itself is no longer a new discovery, here we propose a new angle to interpret the color gradient with the help of our two-dimensional (2-D) modeling method and the $M_{*}$-color relation of ETGs.

\subsection{The Red Sequence and the $M_{*}$-color Relation}

One of the most important scaling relations for ETGs is the tight correlation between their luminosity (or stellar mass) and their color (Sandage \& Visvanathan 1978). This red sequence was first noticed for ETGs in clusters, as it is very prominent in the color-magnitude plane, even without redshift information (e.g., Bower et al. 1992; Gladders et al. 1998). Now, we know that it is a universal relation for all ETGs at $z \approx 0$, regardless of environment (e.g., Bernardi et al. 2003, 2005). Furthermore, the red sequence has merged into the bigger picture of bimodality of galaxies on the $M_{*}$-color plane (Strateva et al. 2001; Baldry et al. 2004). So far, the red sequence has been reliably detected up to $z \approx 1.5$ (e.g., Kodama et al. 1998; Tanaka et al. 2005; Cassata et al. 2007; Mei et al. 2009; Nicol et al. 2011; Fritz et al. 2014), and possibly higher (e.g., Kriek 2008; Whitaker et al. 2010). The slope of the red sequence seems to not evolve much since $z \approx 1.0$ (e.g., Mei et al. 2009; Fritz et al. 2014). The positive slope of the relation suggests that more massive ETGs tend to have higher average metallicity (also older luminosity-weighted age and higher $\alpha$-element enrichment; e.g., Faber 1973; Kodama et al. 1999; Bernardi et al. 2006; Graves et al. 2009). Meanwhile, the evolution of the red sequence from $z \approx 1$ to 0 is consistent with passive evolution of an old stellar population (e.g., Bell et al. 2004; Fritz et al. 2014). During the same period, the stellar mass on the red sequence has doubled (e.g., Faber et al. 2007). Based on these observations, it would seem that massive ETGs most likely evolve along the red sequence during the last 8-9 Gyr after internal star formation has been quenched early and quickly, although the detailed evolutionary track (e.g., Faber et al. 2007; Schawinski et al. 2014) and mechanisms (e.g., Bernardi et al. 2011) still need to be elucidated. Under the two-phase scenario, a significant fraction of mass growth during the second phase should be dominated by mergers with other galaxies that have low star formation. Hence, dry mergers, both minor and major ones, are

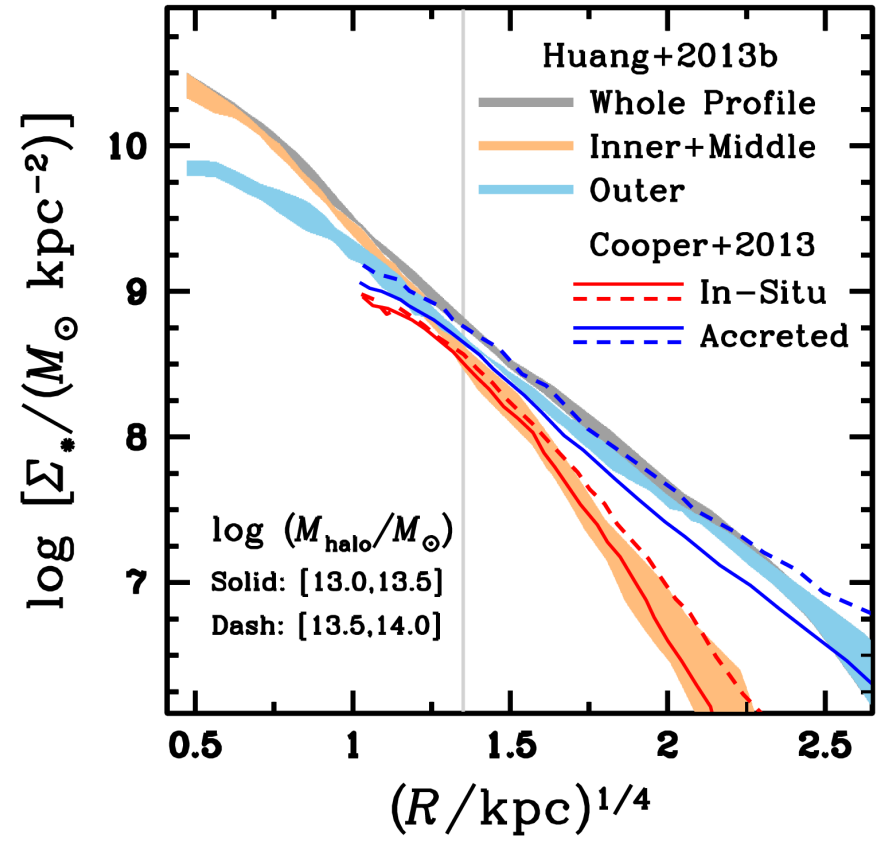

FIG. 1.- Comparison between the median stellar mass density profiles of the inner (orange shaded region) and outer (blue shaded region) photometric components of nearby massive ellipticals (Huang et al. 2013b), and the median profiles of the in-situ (red lines) and accreted (blue lines) stellar components of model massive galaxies (Cooper et al. 2013). Two halo mass bins are selected: $13.0 \leq \log \left(M_{\text {halo }} / M_{\odot}\right)<13.5$ (solid lines) and $13.5 \leq \log \left(M_{\text {halo }} / M_{\odot}\right)<14.0$ (dashed lines). Profiles from simulation are more reliable at radii larger than the scale indicated by the vertical grey line. We account for the difference in the choice of IMF used to estimate stellar mass. The profiles from Cooper et al. (2013) were derived assuming a Chabrier (2001) IMF, while the ones from Huang et al. (2013b) chose the default "diet-Salpeter" IMF from Bell et al. (2001); for a rough comparison, a consistent offset of +0.2 dex was added to the former.

crucial to the evolution of massive galaxies on the red sequence (e.g., Bernardi et al. 2011).

Recent works by Huang et al. (2013a, b) clearly demonstrated that nearby massive ellipticals can be generally decomposed into two parts: a smaller, denser core (inner component) plus an extended stellar envelope (outer component). The properties of the components indicate that they are in line with predictions of the two-phase scenario. If, indeed, more recent merger events were dominated by minor mergers and the stellar contents of these smaller systems are mainly redistributed out to large radii (e.g., Hilz et al. 2012, 2013), then the color and luminosity of the outer component, properly separated from the the core, can provide an estimate on the average mass and number of minor mergers via the $M_{*}$-color relation, as illustrated by the cartoon in Figure 1. Here, we demonstrate the feasibility of this novel approach by extending our 2-D image decomposition technique of Huang et al. (2013a) to multiple filters, and show that this is a promising tool for studying the merger history of ETGs.

The paper is organized as follows. Section 2 gives a brief overview of the sample of ellipticals and the photometric data. We will also briefly describe the 2-D image decomposition method and the main results from $V$-band models. Section 3 provides details about the methods we used to extend current models to other filters and describes the estimation of the $M_{*}$ color relation for nearby ETGs. The main results are summarized in Section 4. Section 5 briefly discusses the assumptions adopted in this work and possible future applications, ending with a summary in Section 6. 
Within this work, we assume $H_{0}=70 \mathrm{~km} \mathrm{~s}^{-1} \mathrm{Mpc}^{-1}, \Omega_{m}=$ 0.27 , and $\Omega_{\Lambda}=0.73$.

\section{PHOTOMETRIC DATA OF NEARBY MASSIVE ELLIPTICALS}

This work uses the multi-band optical images of nearby ellipticals from the Carnegie-Irvine Galaxy Survey (CGS; Ho et al. 2011, Paper I). CGS is a photometric survey of 605 bright $\left(B_{T}<12.9 \mathrm{mag}\right.$ ), nearby (median $D_{L}=24.9 \mathrm{Mpc}$ ) galaxies in the southern sky $\left(\delta<0^{\circ}\right)$. It was designed to provide an optical baseline sample to study in detail the structure of nearby galaxies with different morphologies. The observations were made using the 100-inch du Pont telescope at Las Campanas Observatory to provide high-quality $B V R I$ images. The images have a size of $8.9 \times 8.9$ and a pixel scale of $0 .{ }^{\prime \prime} 259$. Seeing conditions better than $\sim 1^{\prime \prime}$ were achieved for most of the observations. The data were carefully reduced in a standard manner, as described in Paper I and Li et al. (2011, Paper II).

The morphology information for CGS galaxies was initially extracted from the Third Reference Catalogue of Bright Galaxies (de Vaucouleurs et al. 1991), and later visually examined by the authors. In general, $\sim 100$ ellipticals are included in CGS. We managed to perform reliable image decomposition for 94 of them using their $V$-band images (Huang et al. 2013a, Paper III). In this work, we fully take advantage of these existing multi-component models to explore our proposed method for estimating the average merger mass ratio of nearby elliptical galaxies.

As a survey of very nearby galaxies using a relatively small CCD camera, the CGS data cannot compete with large imaging surveys (e.g., SDSS) in terms of sample size and accuracy in photometric calibration. However, the greater image depth, higher spatial resolution, and better seeing conditions provided by CGS are crucial for reliable photometric modeling. Due to the size of the CCD and the extended nature of the ellipticals, an accurate estimate of the sky background is nontrivial (in fact, surveys like the SDSS also suffer from over-subtraction of sky around bright ellipticals; see Blanton et al. 2011). Meanwhile, slightly less than half of the CGS ellipticals were observed under non-ideal photometric conditions. The calibration of these images is less accurate than others, which leads to slightly larger photometric errors. However, we will show later that these data are sufficient to demonstrate the potential of our proposed method.

In Paper III, we performed careful 2-D multi-component image decomposition on the $V$-band images of 94 CGS ellipticals, using the code GALFIT (Peng et al. 2002, 2010). Contrary to common perception, we found that the 2-D surface brightness distribution of nearby ellipticals cannot be described by a single Sérsic (1968) function. All the sources require a multicomponent photometric model. In particular, the photometric models of 70 out of 94 ellipticals have three Sérsic components: (1) a compact central component with effective radius $R_{e} \lesssim 1$ kpc comprising a small fraction of the light $(f \approx 0.1-0.15)$; (2) an intermediate-scale component with $R_{e} \approx 2.5 \mathrm{kpc}$ and $f \approx 0.2-0.25$; and (3) an extended envelope with $R_{e} \approx 10 \mathrm{kpc}$ and $f \approx 0.6$, which is also moderately more flattened than the interior parts.

These models were constructed in a purely empirical manner with no assumed physical meaning assigned to any component. We started from a simple single-Sérsic model, and gradually built up the complexity by introducing more Sérsic components with reasonable constraints, until certain standards were reached. In Paper III, we discussed at length the idea of a "good photometric model." We concluded that, even for ellipticals, a model that follows the detailed 2-D surface brightness distribution is necessary to provide a complete picture of their structures. More than that, we also demonstrated that the locations of the individual sub-components on the photometric projections of the fundamental plane are not only reasonable, but also physically meaningful. The relevant procedures of model building will be described in the next section. Paper III provides more details of our image decomposition procedure.

In Huang et al. (2013b), we further compared these threeSérsic models of nearby massive ellipticals with observations of high- $z$ compact, quiescen ${ }^{8}$ ETGs. The results strongly suggested that a hypothetical structure formed by the combination of the central and intermediate components (referred to collectively as the "inner" component in the rest of the paper) resembles the ETGs at $z \approx 1.5$ in many aspects, including the distribution on the $M_{*}$-size plane and the average mass density profiles outside the central kpc. This evidence supports the idea that the inner part of nearby massive ellipticals can be regarded as the evolutionary descendants of the compact, quiescent galaxies formed at higher redshift under highly dissipative conditions. On the other hand, the extended envelope we isolated from nearby ellipticals (referred to as the "outer" component here) follows a different $M_{*}$-size relation with larger scatter. Compared to the inner component, we also noticed that there is a much more significant correlation between the size of the outer component and the total stellar mass (see Fig. 3 of Huang et al. $2013 \mathrm{~b}$ ), which is predicted by the two-phase scenario (Oser et al. 2010). These pieces of evidence all indicate that the outer component can be seen as the by-product of non-dissipative, minor mergers.

Although the anatomy of nearby massive ellipticals is consistent with the expectations of the two-phase evolution model, it is still difficult to directly compare observations with simulations due to limitations on both sides. In Cooper et al. (2013), the particle-tagging technique was combined with semi-analytic galaxy formation models (Guo et al. 2011) to predict the $M_{*}$ density profiles of massive galaxies in the Millennium II simulation (Boylan-Kolchin et al. 2009). The authors separate the stellar component formed through in-situ star formation from material accreted through mergers. Figure 2 compares the median $M_{*}$ density profiles of the inner and outer components of nearby massive ellipticals $\left(M_{*} \geq 10^{11.15} M_{\odot}\right)$ from Huang et al. (2013b) with the average profiles of the insitu and accreted components of simulated galaxies, in two relevant halo mass bins, from Cooper et al. (2013). The similarity is striking in regions resolved in the simulations. It is still too early to think that all observations can be perfectly explained, but we are motivated to apply our 2-D models to extract more physical insights.

\section{MULTI-COMPONENT DECOMPOSITION IN $B$ AND $R$ BANDS}

\subsection{Basic Ideas}

In the ideal situation, multi-band images of the same galaxy can be modeled simultaneously under certain constraints. Recently, this approach was realized through the efforts from the MegaMorph team (Häußler et al. 2013; Vika et al. 2013, 2014) and has been applied to a large sample of galaxies (e.g., Vulcani et al. 2014). A similar approach has also been used to sepa-

\footnotetext{
${ }^{8}$ In this work, "quiescent" means that the galaxy has very low star formation rate and does not contain a strong active nucleus.
} 

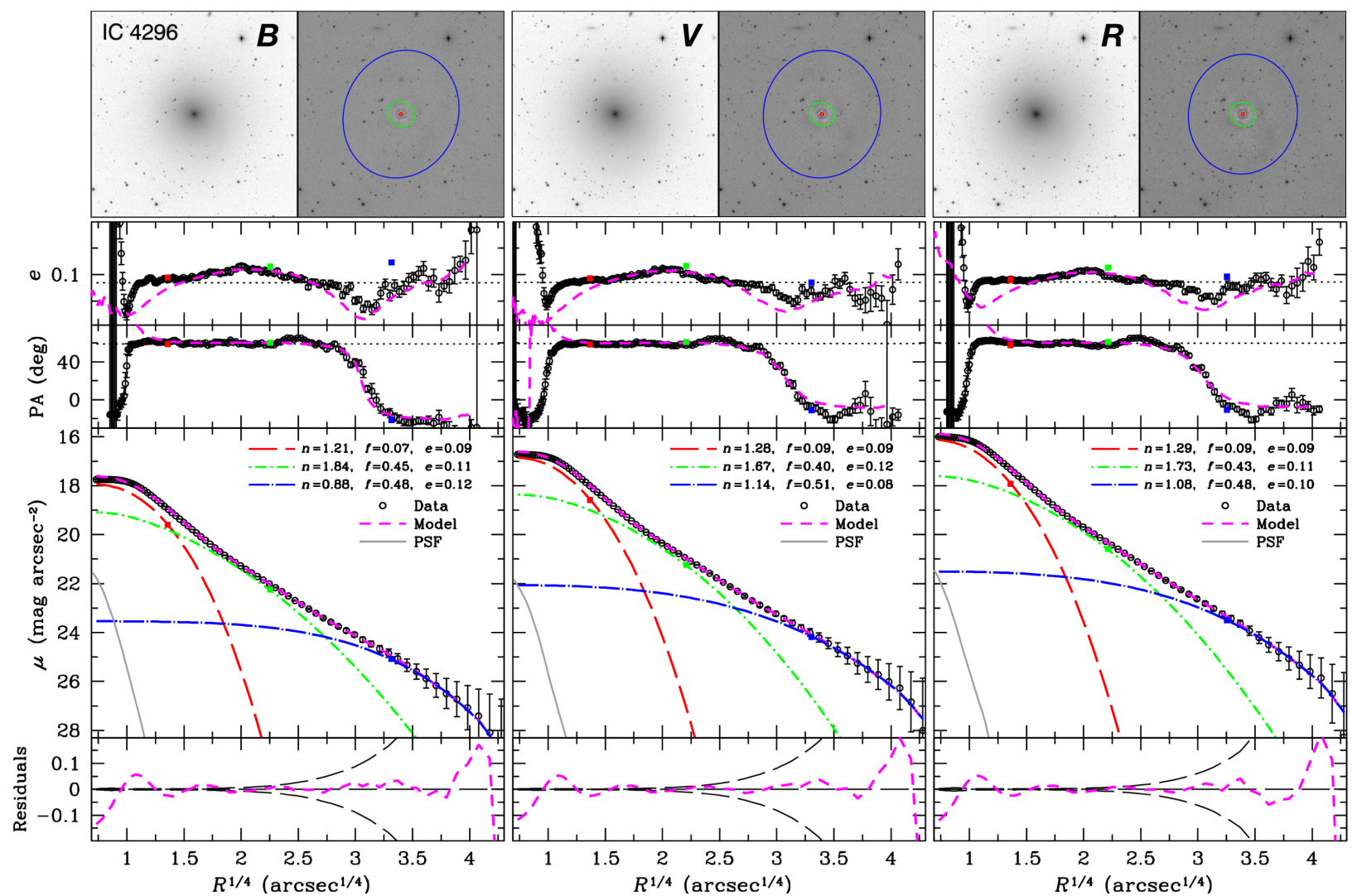

FIG. 3. - Comparison of the photometric decomposition of independently constructed three-Sérsic models for IC 4296 in the $B$ (left), $V$ (middle), and $R$ (right) band. The original image of the galaxy and the residual image (after subtracting the best-fit 2-D model) are displayed in the upper panels. For the 1-D information, the profiles for ellipticity, position angle, surface brightness, and residuals are shown from top to bottom. The observed profiles along with their uncertainties are shown in black data points, while the profiles for the integrated models are shown as a pink dashed line. The surface brightness profile for each Sérsic component is displayed in a different color; the same color scheme is used to trace the half-light radius in the residual images in the upper panels. The surface brightness profile of the PSF model in each filter is also shown (with arbitrary flux zero point). For each component, we list the best-fit Sérsic index $n$, light fraction $f$, and ellipticity $e$.

rate active galactic nuclei from their hosts (e.g., Bennert et al. 2011). However, these methods so far only provide relatively simple modeling options. Moreover, these techniques are still very time-consuming to apply to large images (in the case of CGS, $2048 \times 2048$ pixels). In this work, we developed a very straightforward approach that provides suitable results for our purpose. First, we confirm that consistent three-Sérsic models can be independently obtained in all three filters ( $B V R$ for most of the galaxies) then, we use the three-Sérsic models of a reference filter as blueprint and apply it to the other two bands under reasonable constraints.

Recent works (e.g., Vulcani et al. 2014) that fit single-Sérsic model profiles to ETGs find that their sizes systematically decrease with wavelength, indicative of a negative color gradient. The number of major and minor mergers experienced throughout the lifetime of a galaxy spans a continuum, with ETGs occupying one extreme and disk and bulgeless galaxies lying on the other. In late-type galaxies, the reason that bulges can be differentiated from disks in their light profile and stellar content is that bulges and disks formed along different paths. If the negative color gradient of ETGs is the result of there being different stellar population components, then a multi-component analysis, similar to bulge-to-disk decomposition, might also bear that out. In Huang et al. (2013b), we showed that the inner component has properties that indeed closely match those of red nuggets. If this approach is sound, ideally we expect individual light profile shapes not to change across different filters, only the profile amplitudes. In reality the profile shapes do change due to dust or more complex mix of stellar populations, and this introduces uncertainty into the interpretation.

All ellipticals in Paper III require multiple components, and the majority are best fit by a three-Sérsic photometric model. For the 71 galaxies with reliable $V$-band decomposition, we start by independently building three-Sérsic models for them in the $B$ and $R$ bands, using the method described in Paper III. In our previous work, the $V$-band was selected mainly to minimize the "red halo" effect (e.g., Michard 2002; Wu et al. 2005) of the point-spread function (PSF), and also to take advantage of its relatively lower sky background. To decide the average mass ratio of the mergers using the mass-color relation, we choose to use the colors $B-V$ and $B-R$ over $V-I$, as inclusion of a bluer filter makes them slightly more sensitive to changes in the underlying stellar population (Bell \& de Jong 2001). Hence, the mass-color relations using these two colors have steeper slopes than the one using $V-I$. For the same uncertainty in the average color difference, this leads to slightly smaller uncertainty in the derived mass ratio. As shown in Figure A1 of Paper III, the red halo effect for the PSF is very small in $B$ and $R$. It will still contaminate the color of the low-surface brightness region when its scale is similar to the size of the red halo, but for the ellipticals in our sample, the strongest part of the red halo $\left(10^{\prime \prime}\right.$ to $15^{\prime \prime}$ ) will not fall on the low-surface brightness region. Moreover, with the help of 2-D modeling, we no longer only look at the color at very large radii but the average color of the entire 
component.

Compared to other systematics we face (e.g., photometric calibration, background subtraction), the bias introduced by the red halo effect can be ignored for these three filters (the same cannot be said for the $I$ band, which is why we do not use it in this analysis). If there is still any, it should make the outer component slightly redder. As discussed later, this will not affect our main conclusions.

\subsection{Sky Background Estimation}

Unlike the red halo effect, sky background subtraction is always a difficult issue to treat for massive ellipticals. First and foremost, the extended outer envelope conspires with the limited field-of-view to make estimating accurate sky background difficult. The difference in average sky level and spatial gradient of background among different filters further makes the background subtraction a major source of uncertainty for our models. Under these circumstances, the background level and its gradient (as the field-of-view of our images is not very large, the background can be approximated as a tilted plane; fluctuations of higher order are ignored) become part of the model (see Yoon et al. 2011). In principle, one can fully take this into account by sampling the whole parameter space using a Markov Chain Monte Carlo (MCMC) method (e.g., Yoon et al. 2011; D'Souza et al. 2014). However, such approach is still extremely computationally expensive for large images and multicomponent models ( $\sim 20$ free parameters). In this work, we compute a model-dependent background, in two ways.

1. First, we recalibrate the background of the $B$ and $R$ images using the same method described in the Appendix B of Paper III. In short, we fit the image with a series of models with different number of components and a free background. The average output background values from reasonable models and its scatter are taken as the best guess of the sky level and its uncertainty. During the formal three-Sérsic model fitting, we add a sky component with average intensity fixed at the best guess and free gradient in both the $\mathrm{X}$ and $\mathrm{Y}$ directions. In practice, the spatial gradients of background are very small for our objects.

2. Even after the sky background has been re-calibrated, when we apply a three-Sérsic model from one filter as reference to another filter, we allow the sky value for the new filter to be either fixed or free. For each choice of model, we make two estimates of color based on the stability of the background subtraction.

Even after all these attempts, we still have two sources of uncertainties to worry about. Sky subtraction is still the largest source of uncertainty for our three-Sérsic models. As it significantly affects the surface brightness profiles at large radii, it mainly makes the magnitude and size of the outer component uncertain. Therefore, the accuracy of sky subtraction in both bands basically controls the accuracy of the color difference between the inner and outer components. At the same time, the uncertainty of photometric calibration applies to the image globally. Hence, it is the main source of uncertainty for the absolute value of colors measured in this work, and determines how well we can locate a galaxy on the red sequence. Despite these limitations, it will not stop us from demonstrating the proposed method. As we will show later, the main results are not
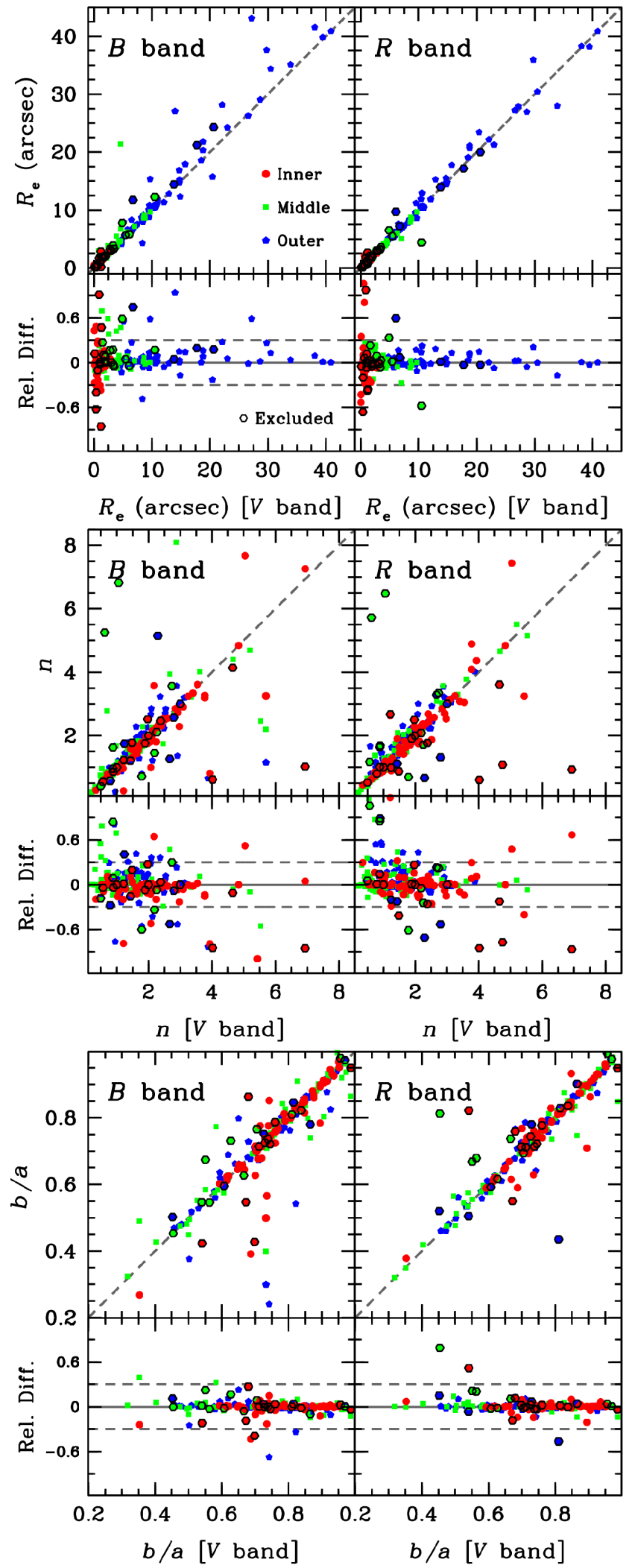

FIG. 4.-Comparison of the effective radius (top), Sérsic index (middle), and axis ratio (bottom) of the three photometric sub-components from the independent $B$-, $V$-, and $R$-band models. The inner, middle, and outer components are displayed using red, green, and blue color. The values for the $V$-band models are used as reference (X axis). A black dashed line indicates the $x=y$ relation in each upper panel. The differences of parameters derived from models in the $B$ and $R$ band and the $V$-band reference models are displayed in the bottom panels. Models that are excluded after this examination are highlighted using black outlines around data points for their components. 


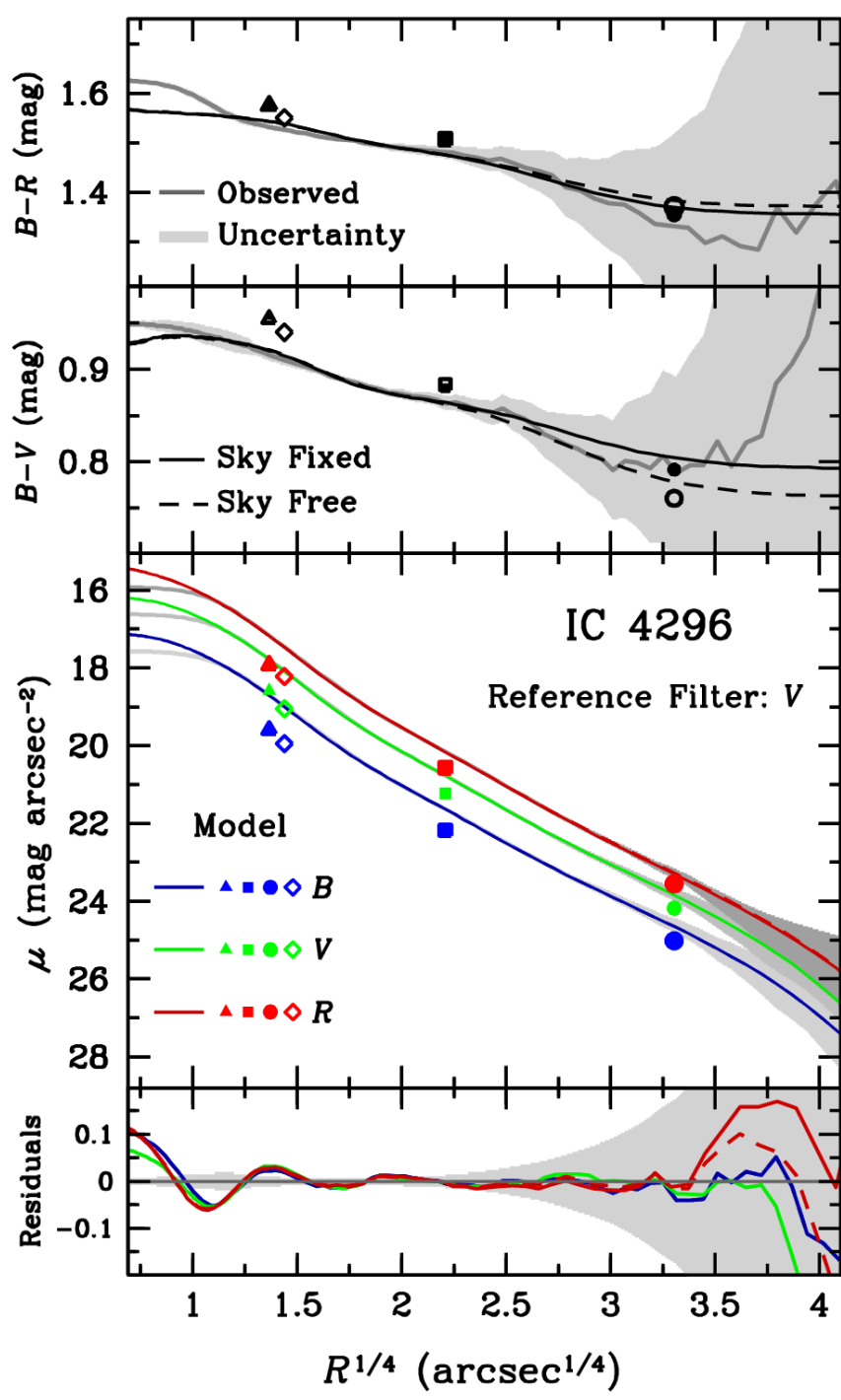

FIG. 5.- 1-D representation of three-component Sérsic models for IC 4296 in the $B, V$, and $R$ bands. From top to bottom, we show the 1-D $B-R$ and $B-V$ color profiles, the surface brightness profiles, and their residual profiles. The observed surface brightness profiles and their uncertainties are shown in slightly different grey shaded regions. The surface brightness profiles for models in $B, V$, and $R$ bands and their residual profiles are shown in different colors. To highlight the properties of all three Sérsic component, different symbols are used to mark the effective radius and effective surface brightness of each component (inner: red; middle: green; outer: blue). The effective radius and the effective surface brightness of the composite inner+outer components are also highlighted using empty diamonds. In the two top panels, the observed 1-D color profiles without correction for PSF convolution are shown in grey solid lines; their uncertainties are shown using a shaded region. The large uncertainties at large radii are partly due to the inclusion of uncertainties in sky background estimation. The color profiles of the models are shown in black lines after corrected for PSF convolution. Two different models are displayed here: fixed sky background (solid line) and free background (dashed line). Similar to the surface brightness plot, the color of each component in the models are highlighted at its effective radius. Filled symbols are used for fixed sky, and open ones are for free sky.

affected. In the future, this situation will be improved by using images with larger sky coverage and more advanced methods in image modeling.

\subsection{Procedures for Model Fitting}

Using the updated sky background value, we first try to independently build a three-Sérsic model in the $B$ and $R$ bands. The PSF model and object mask are prepared exactly as for the $V$-band images in Paper III. To make these models more objective, we do not start from best-fit parameters from the $V$ band. Instead, initial guesses for model parameters are also decided in the way described in Paper III. In general, the results are very encouraging. For most ellipticals with $V$-band threeSersic models, similar models can be obtained in both the $B$ and $R$ bands. Figure 3 shows an example for IC 4296. From the upper panel, we can see that the residuals are very good in all three filters. And judged by the surface brightness profiles and key parameters for the three Sérsic components, the models in all three bands are indeed very consistent. In Figure 4, we further compare the effective radius $\left(R_{e}\right)$, Sérsic index $(n)$, and axis ratio $(b / a)$ for each component in models from the three filters, and show the relative difference using the value from $V$-band as reference. For most galaxies, we have very consistent models in all three bands. In general, the $R$-band models are more similar to the $V$ band than the $B$ band, especially for $R_{e}$ and $b / a$. This is expected, for three reasons: (1) The typical signal-to-noise ratio at large radii and the typical background level is quite different between $B$ and the two redder bands, which can affect $R_{e}$ and $n$ for the outer component. (2) We identified dust features around the center of 21 Es in our sample. However, we will not be able to resolve dust features at smaller scales, as those detected by high-resolution $H S T$ images (e.g., Lauer et al. 2005). Dust naturally has a greater effect at shorter wavelengths. (3) The typical $B$-band PSF is systematically broader than that in the $V$ and $R$ bands. Of course, it is also possible that the differences in $R_{e}$ and $n$ simply reflect some intrinsic wavelength dependence for individual components. We cannot resolve this given the current uncertainties.

After examining these models, we exclude a few galaxies with large differences between their $V$ - and $R$-band results, which may be due to problematic background estimation in either of the filters; $B$-band models are not considered in this process. We mostly focus on the intermediate and outer components during the examination. The large uncertainties for parameters of the central component can be easily affected by dust features, unresolved structure, or tiny PSF errors. As discussed in Paper III, even though the central component tends to have large relative uncertainties (in some cases, the central components are not well-resolved by our observations), they are very much needed to achieve a satisfactory global model. The final sample contains 60 objects.

We use the best-fit three-Sérsic $V$-band models from $\mathrm{Pa}$ per III as reference to generate corresponding models in $B$ and $R$. In light of the main purpose of this work, all parameters for the three Sérsic components are fixed except for their total magnitude (basically a color term is added to each component). As mentioned, a sky component is included, and its value is either fixed to the updated value or set free; sky gradients in both Xand Y-direction are left free for both cases. The background gradients are always quite small for these galaxies. Models without sky gradient essentially show no difference. After the models are built, the color for each component is extracted and the overall color profile is generated; the PSF convolution in both filters is removed before the color profiles are made. In Figure 5, the one-dimensional (1-D) surface brightness profiles of models in all three filters, their residual profiles, and the $B-V$ and $B-R$ color profiles are displayed along with the $R_{e}$, the surface brightness at $R_{e}$, and the color of each components. The residual profiles in the three bands are very good and consistent. More importantly, both the $B-V$ and $B-R$ color profile of our three-Sérsic component models closely follow the 

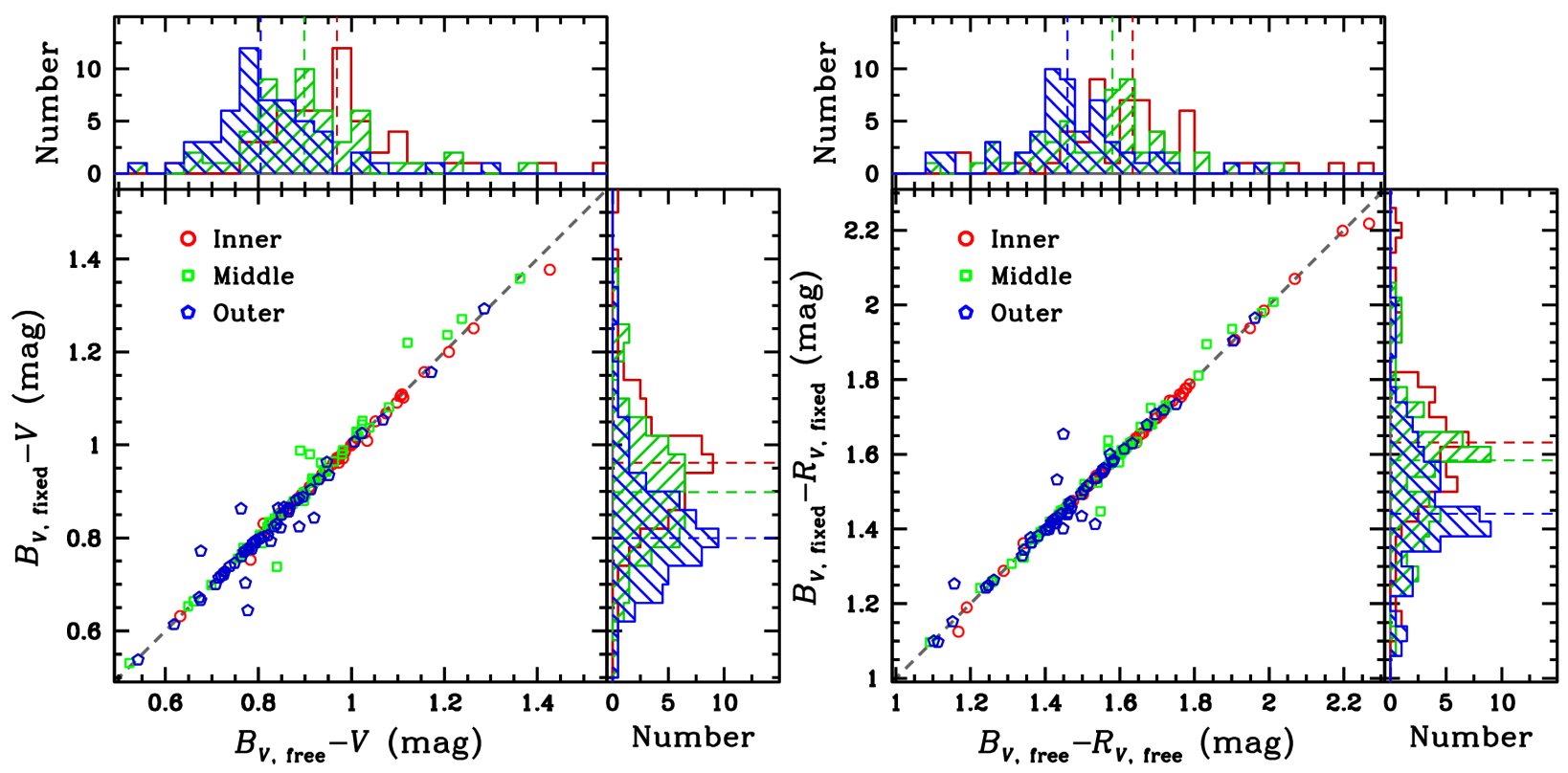

FIG. 6.- Distributions and comparisons of $B-V$ (left) and $B-R$ (right) colors of the inner (red circle), middle (green square), and outer (blue pentagon) components from the sky-free and sky-fixed models. A grey dashed line is shown to indicate the $x=y$ relation. For the histogram, the median color of each component is also highlighted using a dashed line with corresponding color.

observed one. Differences in the innermost region $\left(\sim 1^{\prime \prime}\right)$ are due to different treatment of PSF convolution.

To extract the observed color profile from the data for comparison with the model fits, one has to account for the PSF differences between the different filters. We choose a commonly adopted method: convolve the image in one filter with the PSF model from the other filter before the color profile is extracted. The distinct bump of the $B-R$ profile in the center is likely an artefact, and it has little effect on the color profile of the model. On the other hand, at very large radii, usually close to the edge of the image, the observed $B-V$ color profile sometimes shows a weird upward "U-shape" feature, which is unlikely to be real. As discussed in Section 3.1, it is also not caused by the PSF red halo effect, considering its large radius, but is probably due to slight over-subtraction of background in the $V$ band. Due to the large uncertainty of the background in $V$, the color of the outer component also clearly varies with fixed or free background. In fact, this is an inherent weakness of the 1-D color profile analysis, as it is extremely sensitive to not only the structure of the PSF but also the accuracy of background subtraction. Meanwhile, the color of the outer component from our PSFconvolved 2-D model is much more stable against these issues.

In Figure 6, the $B-V$ and $B-R$ color from the fixed and free sky modeling are compared for each component. The overall agreement is good. Large deviations are mostly seen for the outer component alone, and error in sky determination for at least one of the filters is likely to be the main reason. We also relax the parameter constraints, where both the Sérsic index and total magnitude of each component are free during the fitting. As expected, the extra degrees of freedom lead to minor improvement in the residual, mostly in the central region, which affects the Sérsic index the most. However, it leads to very little to no difference in the inferred average color profile. We therefore stick to the multi-band models where only the magnitude of each component is allowed to vary between the filters. The color information for the sample is summarized in Table 1.

\section{4. $M_{*}$-color Relations}

To interpret the color offset between the inner and outer components, we compare our measurements with the slope of the $M_{*}$-color relation followed by nearby ETGs. According to recent observations, massive quiescent galaxies span a sequence on the $L_{*}$-color or $M_{*}$-color plane, up to $z \approx 1.5$ in both overdense and field-like environments (e.g., Tanaka et al. 2005; Nicol et al. 2011). The slope of the $L_{*}$-color relation for these galaxies appears to have not changed much since $z \approx 1.0$. The evolution of the intercept of the relation favors a quick exponential decline of star formation rate (e.g., Fritz et al. 2014). This is in line with the two-phase formation scenario of massive galaxy evolution.

Figure 7 displays the distributions of all CGS galaxies, according to their morphology, on different $M_{*}$-color and colorcolor planes. Stellar mass for CGS galaxies is derived using the empirical relation between the $B-V$ color and stellar $M / L$ in Bell et al. (2003). To compare with other samples, a -0.2 dex constant offset is applied to make our masses compatible with a Chabrier (2003) stellar initial mass function (IMF); this is a rough approximation, but it has no effect on the slope of $M_{*}$ color relation. The photometric uncertainty of the CGS data leads to larger scatter, but the behavior of each morphology class is reasonable. We will first fit the $M_{*}$-color relation using CGS data alone. The ETG sample comprises all ellipticals and S0s. Under the assumptions of the two-phase formation scenario, most of the mergers during the last phase should be dry (e.g., Tal et al. 2009), and hence it is natural to consider only ETGs, systems that closely track the red sequence.

We adopted the Least Trimmed Squares (LTS) algorithm used in Cappellari et al. (2013), as implemented in the IDL version of the code lts_linefit from Michele Cappellari's website 9 the code is very robust against (the possibly large number of) outliers and can properly account for uncertainties from both axes. Meanwhile, lts_linefit also provides the

\footnotetext{
${ }^{9} \mathrm{http} / / / \mathrm{www}$-astro.physics.ox.ac.uk/ mxc/software/
} 

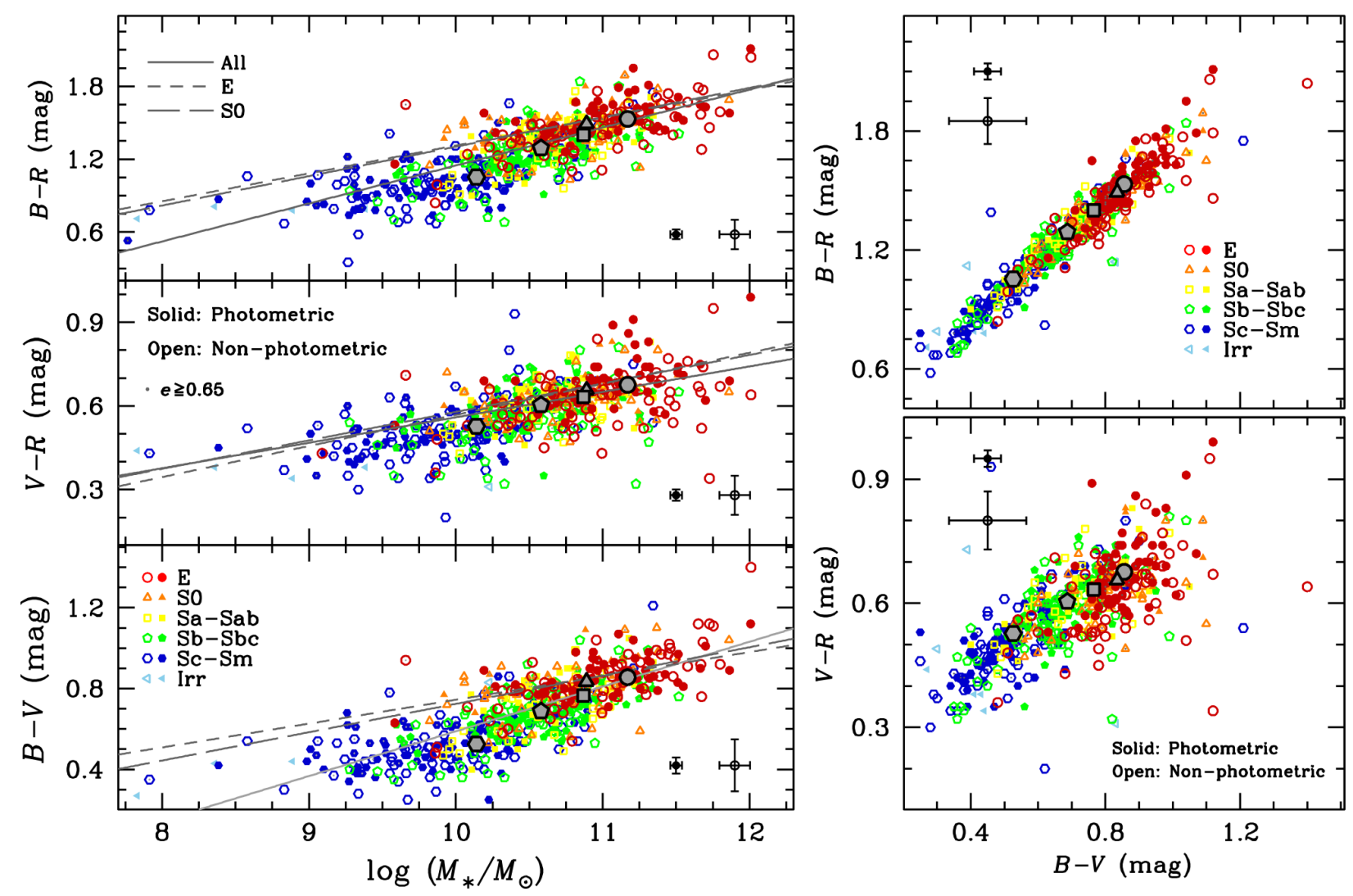

FIG. 7.- The $M_{*}$-color and color-color relations for all CGS galaxies. On the left panel, from bottom to top, the $M_{*}$-color relations for $B-V, V-R$, and $B-R$ colors are displayed. On the right side, the relations between $B-V$ and $B-R$ (top) and $V-R$ (bottom) colors are shown. Different colors and symbols are used for galaxies with different morphologies, as shown in the legends. Filled and open symbols are used to separate galaxies observed under photometric and non-photometric conditions, respectively. The median stellar mass and optical color of each morphological type is shown using large grey symbol. Typical errors for the photometric and non-photometric subsamples are shown on each plot. On all $M_{*}$-color relations, three different lines highlight the general mass dependence for the ellipticals (dashed line), S0s (long-dashed line), and the whole sample (solid line) using simple linear fitting results.

option to use linmix_err by Kelly (2007) to derive the bestfit relation and its uncertainty. We use 10,000 MCMC sampling to get the posterior distribution of parameters in linmix_err. To reduce the influence from outliers, 3- $\sigma$ clipping is performed before the fitting. We also impose a lower stellar mass limit of $10^{9.9} M_{\odot}$ to exclude a few low-mass galaxies with suspicious classification. For CGS data, the errors on the colors and stellar mass are all dominated by the systematic uncertainties from photometric calibration. These errors are clearly highly correlated due to our photometry-based approach to derive stellar mass. The results are shown as part of Figure 8. For the $B-V$ color, the best-fit result is

$$
(B-V)=(0.119 \pm 0.015) \times \log M_{*}-(0.450 \pm 0.160),
$$

and the corresponding relation for the $B-R$ color is

$$
(B-R)=(0.176 \pm 0.030) \times \log M_{*}-(0.410 \pm 0.330) .
$$

As expected, the CGS data result in large uncertainties on the slopes and intercepts of the above relations. Also, lts_linefit results without invoking linmix_err are consistent with above ones.

To ensure that the derived slopes are useful, we seek help from the SDSS, as it provides a more complete, much larger sample of nearby galaxies with uniformly calibrated photometry. Sérsic-fitting photometry of galaxies within $0.05 \leq z \leq 0.07$ was selected from the catalog of Simard et al. (2011). This redshift range ensures that galaxies are nearby enough to be well resolved and can be compared with our CGS sample, while not so nearby in order that the high-mass population can be sampled. We convert the extinction- and $K$-corrected $g-r$ color, derived from Sérsic fitting, to $B-V$ and $B-R$ colors using the empirical equations from Lupton et al. (2005).

This sample is further cross-matched with the spectrophotometric stellar mass estimates based on the Simard et al. models (Mendel et al. 2014). We assign a constant 0.15 dex uncertainty. To obtain a sample of ETGs (ellipticals + S0s), we use the automated morphological classifications from Huertas-Company et al. (2011), who apply a threshold of $p_{\mathrm{E}+\mathrm{S} 0}>0.7$ to isolate likely ETGs. This produces a sample of 16,100 nearby ETGs, which is compared in Figure 8 along with the CGS sample. The general distributions and $M_{*}$ dependence are very similar, though it is likely that a small constant shift in either mass or color direction can make the agreement better. The CGS $B-V$ color is a bit "too blue" when compared with SDSS at similar $M_{*}$, while the CGS $B-R$ color is "too red." This can be easily due to difference in filters and IMF choice. The $M_{*}$-color (converted $B-V$ and $B-R$ ) relations of the SDSS sample are fit in exactly the same way. The best-fit relation for $B-V$ color is

$$
(B-V)=(0.103 \pm 0.001) \times \log M_{*}-(0.234 \pm 0.012),
$$

and for $B-R$ color

$$
(B-R)=(0.162 \pm 0.002) \times \log M_{*}-(0.324 \pm 0.020)
$$



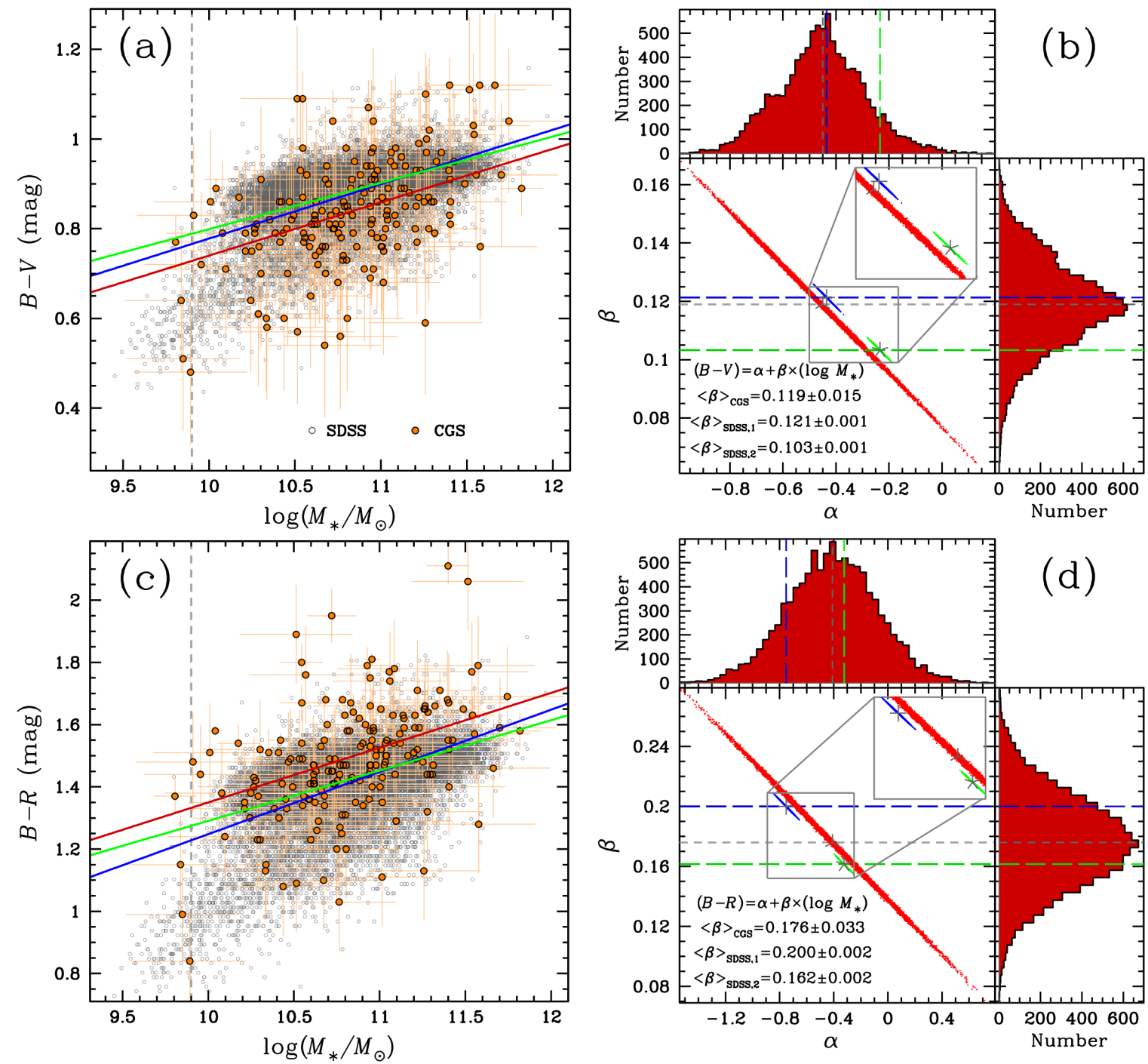

FIG. 8.- The $M_{*}$-color distributions of CGS ETGs and nearby SDSS ETGs, and their best-fit linear relations. Panels (a) and (c) show the distributions of SDSS (grey, open circle) and CGS (orange, filled circle, with error bar) galaxies on the $M_{*}-(B-V)$ and $M_{*}-(B-R)$ planes. A grey dashed line indicates the applied $M_{*}$ cut during the fitting. Panels (b) and (d) display the distributions of slope $(\beta)$ and intercept $(\alpha)$ from the MCMC fitting of the relations for CGS (red), SDSS $p_{\mathrm{E}+\mathrm{S} 0}>0.7$ (green), and SDSS $p_{\mathrm{E}+\mathrm{S} 0}>0.5$ (blue) samples. The final estimation of the slopes are shown on the plots, and also highlighted using different lines. Three solid lines with corresponding colors are displayed on panels (a) and (c) to illustrate the best-fit relations. The red histograms show the posterior distributions of parameters from a $N_{\mathrm{MCMC}}=10000 \mathrm{MCMC}$ run.

As shown in Figure 8, the slopes from SDSS sample are slightly shallower, but can still be seen as consistent with CGS within the uncertainties. The intercepts of the SDSS sample show systematic offsets that confirm the direction of overall shifts between the distributions of these two samples. Although the overall agreement is quite encouraging, especially considering the systematic differences involved, we note that the slopes are quite sensitive to the choice of morphological threshold. If we switch to $p_{\mathrm{E}+\mathrm{S} 0}>0.5$, more $\mathrm{S} 0$ s and other early-type disk galaxies are included, and the slopes for both relations become much steeper: $\beta=0.122$ for $B-V$ and $\beta=0.197$ for $B-R$.

Along with the observed uncertainties, Its_linefit can also account for the intrinsic scatter of the relation, which is important for estimating the uncertainty of the mass ratio we are deriving. However, for the relations based on CGS data, the intrinsic scatter is strongly affected by systematic uncertainties. In the case of the SDSS data, the derived intrinsic scatters are always very small. Although it is known that the red sequence is very tight at the high-mass end, we realize that the uncer- tainty of the slope of the $M_{*}$-color relation is dominated by the choice of which "early-type" galaxies we include in the sample. When more S0 galaxies are included, the color distribution at the low-mass end shifts slightly bluer. This leads to a steeper slope of the $M_{*}$-color relation, as we just showed. Although it is safe to assume that the late-time evolution of massive ellipticals is dominated by non-dissipative mergers, we still know very little about the detailed morphology of the smaller satellites involved in the merger. This uncertainty is interesting, and affects the final estimation of the merger mass ratio. We will return to this point later.

\section{RESULTS}

With the photometric models for the images in the three bands and the $M_{*}$-color relations in hand, the main results of this work are very straightforward to obtain. In the following two sections, we first briefly summarize the color difference between the inner and outer components, and then estimate the average merger mass ratio based on our method. 


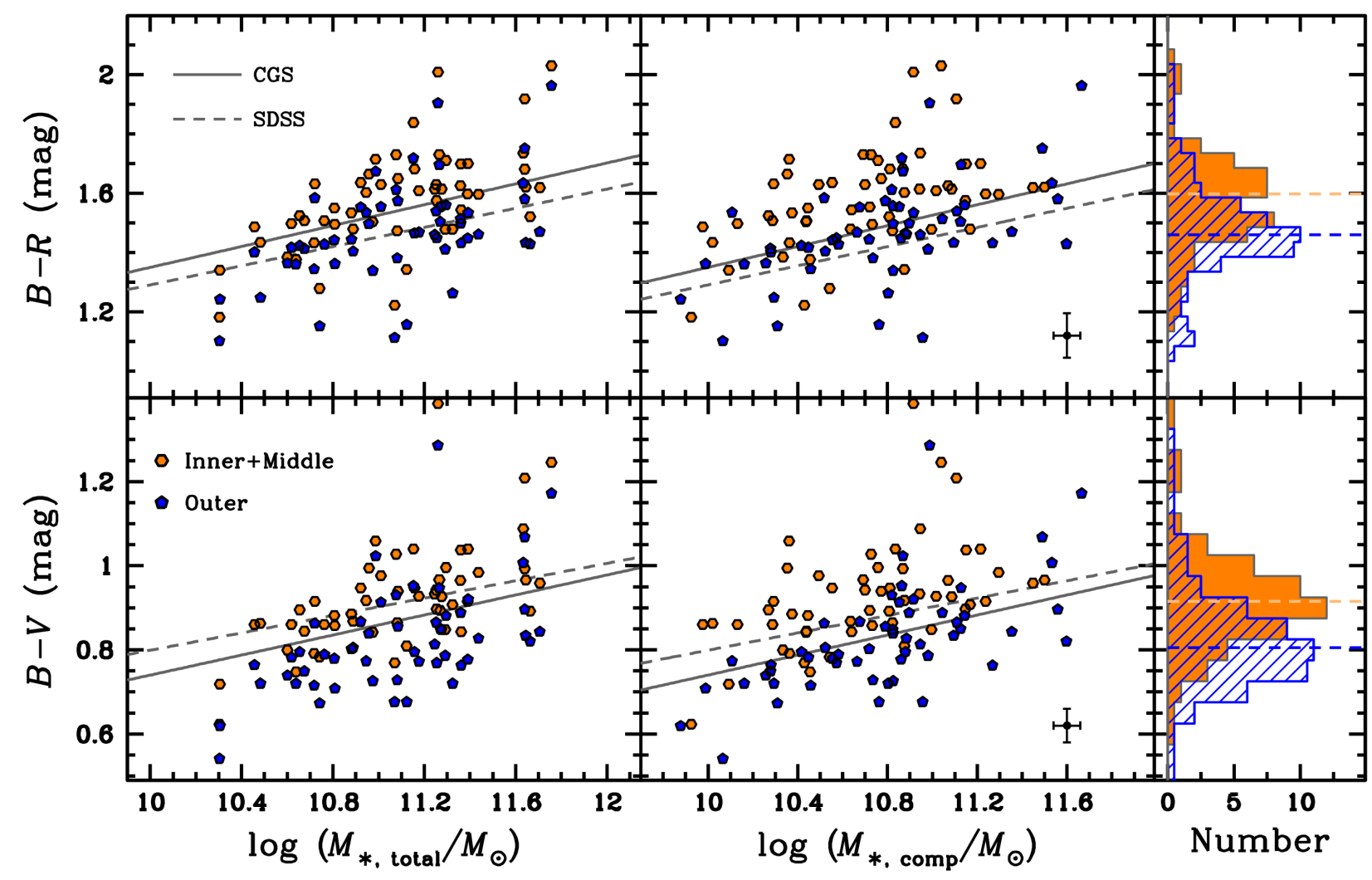

FIG. 9.- Distributions for colors ( $B-R$ : top; $B-V$ : bottom) of the inner (orange) and outer (blue) components, along with their relations with the total stellar mass of the galaxies ( $M_{*, \text { total }}$; left) and the stellar mass of each component $\left(M_{*, \text { comp }}\right.$; middle). The best-fit relations of CGS ETGs (solid line) and SDSS $p_{\mathrm{E}+\mathrm{S} 0}>0.7$ sample (dashed line) are displayed on the left and middle panels. On the histograms on the right, dashed lines with corresponding colors are used to highlight the median value of the color distributions.

\subsection{Color Difference between Inner and Outer Components}

In Section 3, we showed that the well-known negative color gradient in ellipticals can be accurately recovered by a combination of three Sérsic components with very similar properties. This suggests that, as expected, the color of the outer portion should be bluer than the portion at small radii. Here we explore this in detail. First, we compute the integrated color of the hypothetical inner component (central+intermediate) by simply combining their luminosities together. The properties of this structure are known to be similar to those of high- $z$ compact, quiescent galaxies, which are likely to be the progenitors of the massive ellipticals in our sample; they also remind us of the predicted in-situ component in the two-phase scenario for the formation of massive galaxies (Huang et al. (2013b and Figure 1). Meanwhile, the outer component may well represent the accreted stellar envelope. We first examine their color distributions and their behavior on the $M_{*}$-color plane (Figure 9). We consider both the total $M_{*}$ and the stellar mass for each component. We estimate $M_{*}$ of the inner and outer components using their own colors, as derived from models with $V$ band as reference and free sky background in the other filter, in combination with the recipe for $B-V$ color from Bell et al. (2003). The total stellar mass $M_{* \text {,total }}$ is the combination of the inner and outer components. The $M_{*}$ estimated based on the $B-R$ color is systematically higher by $0.08 \pm 0.05 \mathrm{dex}$; the difference does not depend on galaxy luminosity, and the choice of recipe here does not affect any of our results. Recently, Roediger \& Courteau (2015) demonstrate that the $M_{*}$ estimated based on color- $M / L$ relation typically has a random error of $0.10-0.14$ dex, and is not noticeably worse than the one from spectral en- ergy distribution (SED) fitting, given accurate photometry.

In the $M_{* \text {,total }}$-color relations, the outer components are distributed, on average, slightly below the relation defined by CGS ETGs. A few ellipticals on the high-mass end show suspiciously red colors. Two of them (NGC 4760 and NGC 7145) were observed under non-photometric conditions, and hence their colors may be suspicious. In any case, we will rely mostly on the statistical average properties, which should be more robust. When the inner and outer components are placed on the $M_{*}$-color relations using their own stellar mass, they both seem to follow relations that share very similar slopes with the ones defined by the local ETG population. Importantly, the outer components now almost perfectly follow the global $M_{*}$-color relations for ETGs. This is consistent with our assumption that the outer part grows by merging with other galaxies that are already on the red sequence.

The distributions of the color difference between the inner and outer components, and their dependence on $M_{*, \text { total }}$, are shown in Figure 10. For further analysis, we split the sample into high-mass and low-mass subsamples, using the median total stellar mass of $10^{11.10} M_{\odot}$. Median and mean color differences are estimated for them separately (the green and orange points). The high-mass subsample is most relevant for the twophase formation scenario. According to recent works (e.g., Patel et al. 2013), these very massive ellipticals are likely to be the direct descendants of high- $z$ red nuggets. As the most massive systems, they are more likely to have experienced more accretion events. Their photometric properties from our decomposition are also systematically different from those of lower mass objects, being more consistent with theoretical expectations for 


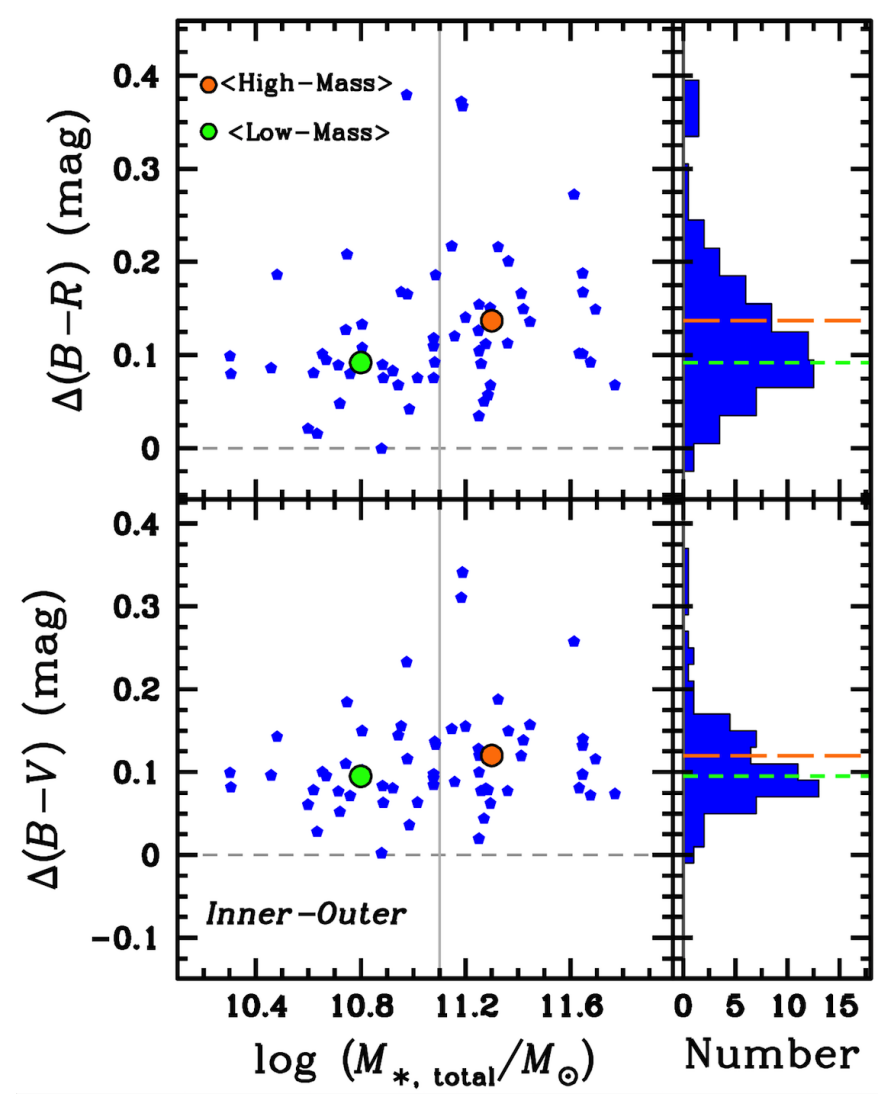

FIG. 10.- Distributions of color difference between inner and outer components and their relations with the total stellar mass. The samples are separated into low-mass and high-mass subsamples using their median value of $M_{* \text {,total }}$ (vertical grey solid line). The median value of $M_{*, \text { total }}$ and color difference for both subsamples are highlighted using green (low-mass) and orange (highmass) filled circles.

the two-phase formation scenario (Paper III and Huang et al. 2013b). The choice of $M_{*}$ boundary is a bit arbitrary, but the mass range for the high-mass subsample turns out to be appropriate. We chose the models with sky parameter left free because they contain fewer extreme outliers, but the sky-fixed models give consistent results.

The median (average) color difference for the whole sample is $\Delta(B-V)=0.10(0.11)$ mag. For the high-mass subsample, the difference is slightly higher, $\Delta(B-V)=0.12(0.13)$ mag for the median (average), whereas for the low-mass subsample $\Delta(B-V)=0.10(0.10) \mathrm{mag}$. The $B-R$ color behaves similarly. The color difference for the high-mass subsample, $\Delta(B-R)=0.14(0.14)$ mag is systematically larger than that for the low-mass subsample, $\Delta(B-R)=0.09(0.10)$ mag. It is worth pointing out that the color difference between subcomponents derived from 2-D models is not equivalent to the color difference within a radius range derived from the 1-D color profile. The two sub-components highly overlap with each other, just as predicted for the in-situ and accreted components in simulations (e.g., Cooper et al. 2013; RodriquezGomez et al. 2015). Therefore, the 1-D color difference across the overlapped region should be smaller than the color difference between the average color of the inner and outer components. In Appendix A, we compare the 1-D color profiles from our 2-D models with previous works, and show that the 1-D color difference based on our models are consistent with the median color profiles of galaxies in the same stellar mass range. The average 1-D color difference between 3 and 30 $\mathrm{kpc}$ (or between 0.5 to 4 times $R_{\mathrm{e}}$ ) is indeed smaller than the $\Delta(B-V)$ and $\Delta(B-R)$ between inner and outer components. Assuming the in-situ and accreted components are reasonably represented by our models, this suggests that one has to go to radius larger than $\sim 30 \mathrm{kpc}$ or $\sim 4 R_{\mathrm{e}}$ to find regions that are completely dominated by the accreted component; at the same time, the contribution of accreted stars to the projected stellar mass density inside $R_{\mathrm{e}}$ still cannot be completely ignored (e.g. Rodriquez-Gomez et al. 2015).

\subsection{Average Merger Mass Ratio}

Finally, we use the inner-outer color difference and the slope of the $M_{*}$-color relations to estimate the luminosity-weighted average merger mass ratio that built the outer envelope. Although the mass assembly history through mergers is a key ingredient for the theory of massive galaxy formation, it is extremely difficult to reconstruct using just the fossil records at $z \sim 0$ : these mergers often involve galaxies in a range of mass ratio, morphology, and stellar population properties; and they happened across a very long time scale $(\sim 8-9 \mathrm{Gyr})$. Therefore, instead of getting into the details of the merging history, we propose to extract a key statistical value of these mergerstheir average mass ratio-using photometric data alone, within the framework of the two-phase formation scenario. The dense stellar systems formed during the first phase (through intense starburst induced by cold gas accretion and gas-rich mergers) become the inner core of nearby massive ETGs. They quenched their star formation long ago through apparently very efficient processes, settled on the red sequence, and evolved along this sequence as they grew more massive through mergers, all the while becoming redder (on average) due to passive evolution of the stellar population. Based on theoretical predictions, most of these mergers should be non-dissipative, and, by number, minor mergers (mass ratio smaller than $1: 3$ or $1: 4$ ) should dominate. Such predictions have gained much recent observational support, such as the color statistics of satellites and tidal features around massive galaxies and the stellar population properties of the outer regions of nearby ellipticals.

To achieve this goal, we need an empirical relation between the stellar mass and an observable property that satisfies at least the following two conditions: (1) the in-situ part of massive galaxies and most of the galaxies that merged into them follow this relation; (2) the shape or slope of the relation does not evolve strongly up to $z \approx 1$. The red sequence of quiescent galaxies, or the $M_{*}$-color relation, is the best candidate we have. As explained above, the non-dissipative nature of most mergers means that the less massive progenitors should be located close to the red sequence when the merger happened. Observations up to $z \approx 1$ suggest that, although the fraction of galaxies on the red sequence, the relative fraction of low- and high- $M_{*}$ galaxies, and the normalization of the relation all show redshift evolution, its slope seems to be very stable (e.g., Mei et al. 2009; Fritz et al. 2014; Cerulo et al. 2016) and does not depend on the environment. These properties suggest that the evolution of the $M_{*}$-color relation is dominated by the aging of stellar population, making it appropriate for our application in this work.

As shown in previous sections, the observed colors of the outer components are systematically bluer than those of the inner components, and are apparently "too blue" for the $M_{*}$ of the system (see left panel of Figure 9). If the in-situ and accreted components are reasonably represented by our inner and outer components, the color offset we find reflects the difference in 


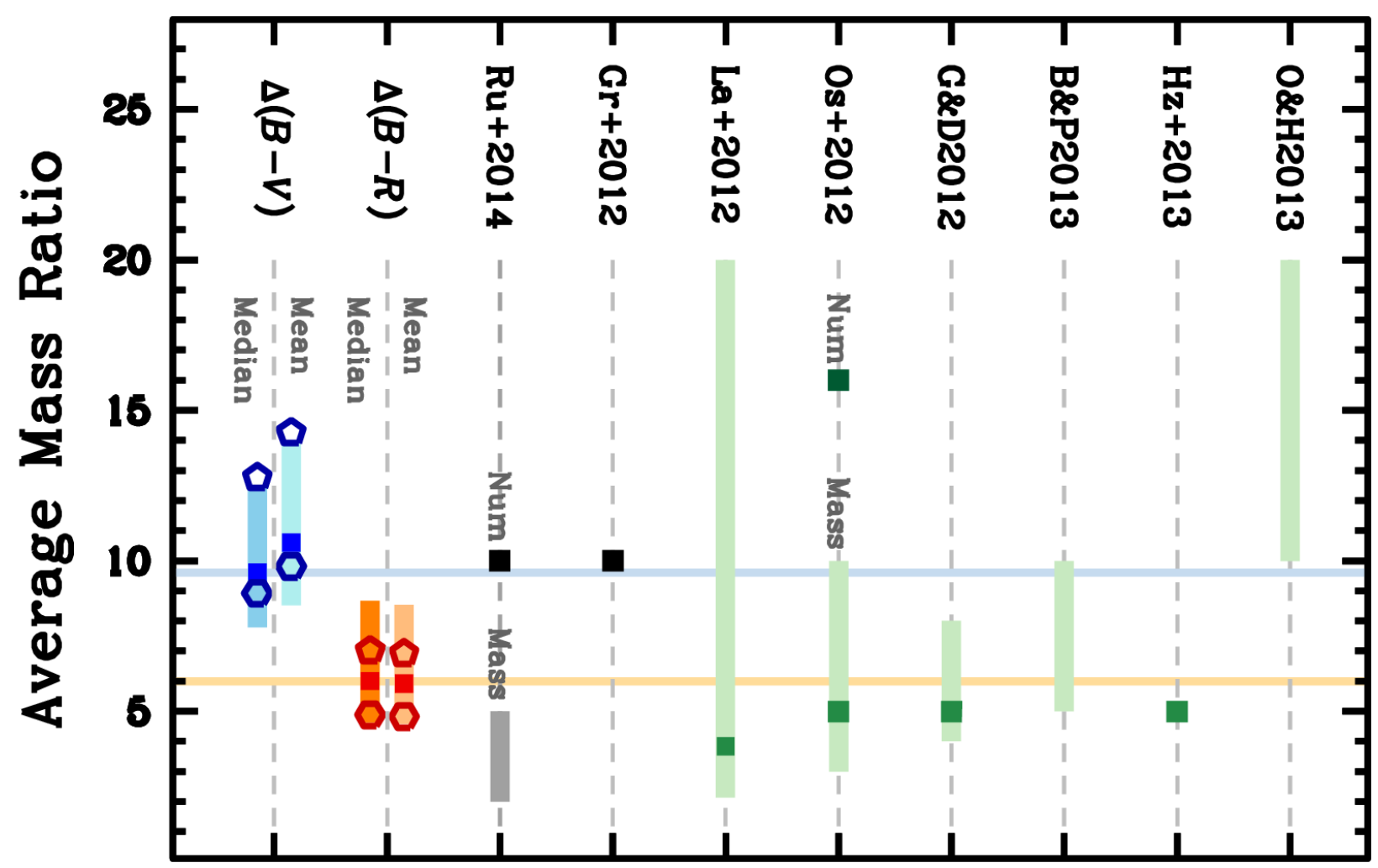

FIG. 11.- The distribution of average stellar mass ratio from this work and from various literature sources. The estimations using $B-V$ (blue) and $B-R$ (red) colors from this work are displayed on the left side, using both the median and mean color difference. Solid squares in darker colors are used for the estimations using the best-fit CGS relations; a lighter, shaded bar illustrates the allowed mass ratio from the $1 \sigma$ uncertainties of the relations. Open pentagons and hexagons with corresponding colors are used for the estimations using the best-fit slopes for the SDSS $p_{\mathrm{E}+\mathrm{SO}}>0.7$ and $>0.5$ samples. The estimations using median color difference and best-fit CGS relations are used as reference. Two horizontal solid lines with slightly lighter colors are displayed to compare with other estimations. Similar estimations in recent observational works are highlighted using grey squares and vertical bars, while estimations from numerical simulations are shown in the same way using different green colors. See the text for detailed explanations of each reference used here. Reference code: Gr+2012 = Greene et al. (2012); $\mathrm{Ru}+2014$ = Ruiz et al. (2014); La+2012 = Lackner et al. (2012); Os+2012 = Oser et al. (2012); G\&D2012 = Gabor \& Davé (2012); Hz+2013 = Hilz et al. (2013); O\&H2013 = Oogi \& Habe (2013); B\&P2013 = Bédorf \& Portegies Zwart (2013).

their average stellar population properties. Using the assumptions discussed above, the color difference can be converted into the mass ratio between the stellar mass of the dense core $\left(M_{*, \text { core }}\right)$ and the typical mass of the satellite galaxy $\left(\left\langle M_{*, \text { sat }}\right\rangle\right)$ using the $M_{*}$-color relations we derived:

$$
\begin{aligned}
\langle\text { Mass Ratio }\rangle & =M_{* \text { core }} /\left\langle M_{*, \text { sat }}\right\rangle \\
& =10^{\left(\left\langle\text {Color }_{\text {inner }}\right\rangle-\langle\text { Color outer }\rangle / \beta\right.},
\end{aligned}
$$

where $\beta$ is the slope of the adopted $M_{*}$-color relation. The definition of mass ratio used here is strongly tied to our assumption of a two-phase formation scenario, and is similar to what has been used in other observational works (e.g., Arnold et al. 2011; Greene et al. 2012; Ruiz et al. 2014) and simulations (e.g., Hilz et al. 2013; Oogi \& Habe 2013).

It is worth reminding the reader here that, in cosmological simulations, the average merger mass ratio is the numberweighted or mass-weighted mean of the instantaneous mass ratio of all the mergers, while under our assumption, we always compare the mass of the merging system with the in-situ core of the main progenitor. At the same time, we assume that all the stars assembled through mergers end up in our outer component. This picture becomes uncertain when major mergers occur, as the dense core of the slightly less massive progenitor may also become part of the inner component. If that were indeed the case, our merger mass ratio should be seen as a lower limit. (This issue will be revisited in the Discussion section.)
Moreover, as our mass ratio estimate is based on the overall stellar population of the outer envelope, it should be considered a luminosity-weighted average, similar to the mass-weighted mean from simulations.

Starting with the color difference between the inner and outer components of the high- $M_{*}$ subsample, we use the best-fit slope of the $M_{*}$-color relations for CGS ETGs, along with their 1- $\sigma$ uncertainties, to estimate the average mass ratio and its associated uncertainty (blue and orange color bars on Figure 11). For the median $\Delta(B-V)$, the mass ratio ranges between $8: 1$ and 12:1, while the range becomes 5:1 to $9: 1$ for the median $\Delta(B-R)$. At the same time, the average mass ratio predicted by the SDSS samples are shown in Figure 11, too.

For the $M_{*}-(B-V)$ relation of the SDSS sample of ETGs defined by $P_{\mathrm{E}+\mathrm{SO}}>0.7$, its shallower slope results in a larger mass ratio $\sim 13: 1$. However, when a slightly different criterion is used to define the ETG sample $\left(P_{\mathrm{E}+\mathrm{S} 0}>0.5\right)$, the slope of the $M_{*}-(B-V)$ relation is more consistent with that for CGS, resulting in a similar average mass ratio. For the $B-R$ color, both SDSS samples predict a similar average mass ratio as CGS. The dependence of the slope on the types of galaxies included in the mass-color relation reminds us another important aspect of our key assumption, which is that the mass-color relation used here should be an appropriate one for most galaxies involved in the assembly history of massive ETGs after $z \sim 1$. Although more and more evidence suggests that non-dissipative merg- 
ers should dominate this process, possible contribution of disk galaxies also plays a role here, and will be discussed in detail in Section 5.2.

As the $M_{*}$-color relation for $B-V$ has shallower slope than that for $B-R$, while the color difference is comparable [median $\Delta(B-V) \approx \Delta(B-R) \simeq 0.1 \mathrm{mag}$ for the high-mass sample], the $B-V$ color difference predicts a somewhat higher average mass ratio than $B-R$ (10:1 versus 5:1). Considering that the average uncertainty of color is roughly the same for $B-V$ and $B-R(R$ band data have slightly better signal-to-noise ratio for massive ETGs), the relation with shallower slope will naturally be more uncertain in predicting the mass ratio. At the same time, since $B-R$ color is more sensitive to the change of stellar mass-tolight ratio of the underlying population (Bell \& de Jong 2001), we expect the $M_{*}-(B-R)$ relation with a steeper slope to have better diagnostic power here. For these reasons, we favor the average mass ratio derived using $B-R$ color. However, such an offset is still a concern, as it may reflect systematic uncertainties. It may also indicate the intrinsic limitation of optical colors for this application. We will discuss this issue further in Section 5.3.

Figure 11 compares our results with estimates from other recent observational and theoretical studies (Section 1), which define the average mass ratio as either number-weighted or $M_{*}$ weighted. Because the average colors we are using are actually luminosity-weighted quantities, the derived average mass ratio using the $M_{*}$-color relation can no longer be seen as $M_{*^{-}}$ weighted. Nevertheless, as both the inner core and outer envelope of massive ellipticals are dominated by a quiescent, old population, their average mass-to-light ratios are not significantly different. When compared with other estimates, our results are still closer to the $M_{*}$-weighted ones than the numberweighted ones. In general, our estimates based on $B-R$ color are reasonably consistent with the average mass ratio predicted by recent simulations (Lackner et al. 2012; Oser et al. 2012; Gabor \& Davé et al. 2012; Bédorf \& Portegies Zwart 2013; Hilz et al. 2013). The high mass ratio suggested by Oogi \& Habe (2013) is number-weighted. In Greene et al. (2012), the average merger ratio is derived in a similar spirit as ours (see their Figures 6 and 7), but based on the equivalent width of the $\mathrm{Mg}_{\mathrm{b}}$ absorption feature and its relation with the velocity dispersion of the galaxy. Their average ratio of $\sim 10: 1$ is consistent with our estimate based on $B-V$ color, but is higher than that based on $B-R$.

The work of Ruiz et al. (2014) uses a very different strategy: the mass ratio statistics of satellites around nearby massive galaxies. Smaller galaxies with 1:2 to 1:5 mass ratio dominate the stellar mass budget of satellites. However, the authors concluded that when these satellites fall into their central host, "the merger channel will be largely dominated by satellites with a mass ratio down to 1:10." Thus, this result also broadly agrees with ours, although we note that the satellite statistics at $z \approx 0$ may not give the full picture of all mergers that occurred during the last $\sim 8-9$ Gyr. Of course, our analysis shares a similar uncertainty, as we are using the $M_{*}$-color relations at $z \approx 0$. More recently, Rodriguez-Gomez et al. (2016) found that in Illustris (Vogelsberger et al. 2014) simulation, roughly $50 \%$ of stars from mergers are contributed by mergers with mass ratio larger than $1: 4 ; \sim 25 \%$ come from mergers with mass ratio between 1:4 and 1:10; the rest $\sim 25 \%$ are from even more minor mergers. A naive estimate of "mass-weighted" merger mass ratio should be around 1:4 to 1:5, which is quite similar to our result using $B-R$ color. As discussed later, it is possible that not all stars accreted through major mergers are located in our outer component, which will make two results even more consistent.

\section{DISCUSSION}

The mass assembly of massive galaxies is dominated by hierarchical merging processes in the last 8-10 Gyr. Although it is not realistic for us to reconstruct their merging history just based on observations at low redshift, the statistical characteristics of such a process may provide useful constraints on the formation scenario. In this work, we present a novel method to estimate the average mass ratio of mergers - an important parameter for the two-phase formation scenario- that built today's massive ETGs. Although the negative color gradient of massive ETGs has been studied for decades, here we propose to explain it in a new context with the help of 2-D modeling. In light of the recent progress in understanding the evolution of massive galaxies, traditional interpretation of 1-D "color gradients" are too oversimplified. Motivated by the "two-phase" formation scenario, we show that the multi-band photometric properties of nearby, massive ellipticals can be well described by the superposition of two sub-components with slightly different colors. After investigating into the size, shape, scaling relations, and average mass profiles of these two components, we find that the outer components in our models can generally represent the accreted stars from all previous accretion events. Assuming most mergers that happened to these massive ellipticals after $z \approx 2$ are non-dissipative, we use the $M_{*}$-color relation of quiescent galaxies to infer the mass ratio between the in-situ part of current massive galaxies and red galaxies that share the same average optical color with the accreted component. We demonstrate that it is possible to extract crucial information regarding the assembly history of massive galaxies without getting into the complex details of stellar population models. Our results are consistent with the expectation that minor mergers are more important for the build up of the extended envelope of massive ellipticals, and also consistent with studies based on different methods (e.g., photometric: La Barbera et al. 2012; spectroscopic: Greene et al. 2013). All that said, we still need to emphasize here that our analysis alone cannot lift the degeneracy between the number of mergers and the average merger mass ratio. We cannot provide more information about the merging history other than a luminosity-weighted average merger mass ratio. The real merger mass ratio certainly does not have a normal distribution centered on such an average value (major mergers contribute more in mass, while minor ones contribute more in absolute number).

\subsection{Color Gradients for the In-situ and Accreted Components}

The most important assumption in this study is that the color gradient arises from two different stellar populations, tracing the in-situ and accreted components, which can be separated spatially. This is supported by recent simulations (e.g., Hirschmann et al. 2015). Although the extreme outer envelopes of nearby ellipticals sometimes exhibit decoupled kinematics and stellar population properties (e.g., Coccato et al. 2010, 2013) the observed color gradient of these galaxies are mostly smooth and featureless, which makes them easily reproduced by superposition of two sub-components with constant color. However, it does not rule out the possibility that both the in-situ and accreted components can have their own color (and underlying stellar population) gradients. Formed through highly dissipative processes, the intrinsic color gradient of the in-situ (in 
our work, inner) component may tell us more about their formation history. If no major merger has taken place, the relatively steep color gradient from intense star formation induced by efficient cold gas accretion should have been preserved to $z \approx 0$. However, one major merger alone may be able to erase such steep gradients. And, for the accreted component, recent simulations (e.g., Rodriguez-Gomez et al. 2016) suggest that accreted stars from different mergers are redistributed to increasingly larger radii in the order of decreasing merger mass ratio. This could naturally lead to color gradient in the accreted component. Of course, during moderate or high mass ratio mergers, a certain level of mixing must occur, which leads to smoother color gradient when the inner and outer parts of these galaxies are assembled.

Although it is already possible to discuss these properties using simulations, observational confirmation is still extremely difficult. In practice, we could give more freedom to both inner and outer components (e.g., free Sérsic index or effective radius) to simulate the color gradient of each component, but this will lead to more complicated internal degeneracies. Meanwhile, if reliable photometry is available at the low-surface brightness, outer regions of these galaxies $\left(>4 R_{\mathrm{e}}\right)$, it is still possible to study the color gradient of accreted stars alone under the assumption that in-situ stars make little contribution there. However, this requires very accurate background subtraction in multiple bands, which makes the data reduction very challenging.

Another issue is the definition of in-situ and accreted components. In simulations, stars formed within the halo of the main progenitor (the more massive one) is often referred to as the in-situ part, while everything else is accreted. In reality, we know that most massive ellipticals experienced major mergers, in which the "less massive" progenitor itself can be quite massive and have its own in-situ and accreted components. In simulations of non-dissipative major mergers, the rank-order of binding energy (radius) is often preserved (Barnes 1988; Hopkins et al. 2009b), which means the in-situ (inner, dense) components of both progenitors will remain as the inner component of the final merger remnant. Under this picture, our inner component may better represent the combined in-situ stars from all progenitors involved in past major mergers. If this is true, it means that the color difference between the inner and outer components could bias the average merger mass ratio toward lower values. It would be interesting to test this idea using simulations.

\subsection{Uncertainty of the Slope of Mass-Color Relation}

As shown earlier, there is still uncertainty in the slope of the mass-color relation, and it directly impacts the merger mass ratio we measured. Such uncertainty highlights another issue in our assumption: we should use the $M_{*}$-color relation followed by the in-situ components and most galaxies involved in the merger history. Disk galaxies must also contribute to the build-up of stellar envelope of massive ellipticals, and they follow a steeper $M_{*}$-color relation. Considering the distribution of their stellar mass, the inclusion of more disk galaxies makes the overall $M_{*}$-color relation steeper, and has larger scatter at the low-mass end. This changes the average merger mass ratio toward higher values. Other than the S0-like, red disk galaxies we considered, there are other bluer, star-forming disk galaxies that live below the red sequence. However, as the outer part of elliptical galaxies is not younger than the inner core, we think that the impact of star-forming disk galaxies in our analysis is negligible.

Besides this uncertainty, we should also point out that systematic change of stellar metallicity is likely the main reason behind both the color gradient in each galaxy and the slope of the red sequence. It is known that the relation between mass and stellar metallicity shows a larger scatter at the low mass end (Gallazzi et al. 2005; Panter et al. 2008; González Delgado et al. 2014). Although the conversion between optical color and metallicity is complex, we cannot rule out the possibility that our $M_{*}$-color relation does not capture the low-metallicity, quiescent galaxies that are significant bluer than the red sequence.

The merging history of massive galaxies can be intrinsically complex in nature, but we want to emphasize here that our goal is to study the luminosity-weighted average properties of all accreted stars, and we think that we can still safely assume that the average behavior of mergers in the last 8-9 Gyr can be approximated using galaxies on the red sequence. It is certainly worth looking into these details using simulations and observations at higher redshift.

\subsection{Details about the Minor Mergers}

Another assumption of our method is that dry, minor mergers dominate the mass growth of massive ellipticals in the second phase of their evolution. To be more specific, we assume that most mergers are between a massive quiescent galaxy and its less massive satellites, and that by the time the merger occurred, the satellites have already become quiescent galaxies on the red sequence. This is a strong assumption, considering that these mergers span a large range in mass ratio and happened within a very long timescale (8-9 Gyr). In general, such a scenario is supported by simulations, but we still need more observational constraints. In the nearby Universe, merger statistics (e.g., Ruiz et al. 2014) do support the notion that minor mergers dominate the merging channel of massive galaxies. Moreover, Ruiz et al. (2015) suggests that massive ellipticals tend to have more satellites, both in number and in stellar mass fraction, than galaxies of other morphologies. At the same time, many different studies also highlight the importance of major mergers (or, at least, "massive" minor mergers) in shaping massive galaxies, especially the contribution to the total stellar mass of the accreted component, at both low and high redshifts (e.g., Ferreras et al. 2014; Kaviraj et al. 2014; Ruiz et al. 2014). Massive halos and their central galaxies tend to have complex merger histories that might not be well described by an average merger mass ratio. Nevertheless, the average mass ratio is a convenient, if blunt, tool for comparison with simulations.

Regarding the notion that the mergers were non-dissipative, there are several lines of supporting evidence. In the local Universe most tidal features around massive ETGs are consistent with an origin in dry mergers (e.g., Tal et al. 2009; Gu et al. 2013). Satellite statistics based on SDSS suggest that most satellites around massive galaxies are red, regardless of the environment (e.g., Hansen et al. 2009 for groups and clusters; Wang et al. 2014 for isolated bright galaxies). At higher redshift, similar studies also have shown that mergers do not introduce a significant younger population of stars (e.g., Ferreras et al. 2014). All these lines of evidence indeed support the idea that massive galaxies mostly grow along the red sequence by merging with galaxies that are already on the same sequence, as suggested by theoretical work (e.g., Skelton et al. 2009). However, just dry mergers alone may not be able to 
explain all the structural evolution and scaling relations of massive galaxies since $z \approx 1$ (e.g., Sonnenfeld et al. 2014). It seems that a small fraction of stars formed in accreted gas is still necessary. However, the detailed impact of such a process on the color distribution is still not clear.

Just knowing that most mergers are dry is not enough. At the same mass ratio, accreting a compact elliptical galaxy may result in different stellar distribution compared with accreting an S0-like galaxy. Hence, it is crucial to learn more about the morphology, structure, and stellar population of satellites involved in these mergers. Greene et al. (2013), through detailed comparison of chemical abundances, concluded that the envelope of massive galaxies are more consistent with stars in quiescent disk galaxies. Here, just using optical color, we actually see the hint of a similar story. As we tried to fit the $M_{*}$-color relation using both CGS and SDSS data, we noticed that the slope of the red sequence becomes shallower when only ellipticals are included. Such shallower slope would result in much smaller average merger ratio, hence leading to an unrealistically large number of minor mergers. This tension disappears when some S0s are included in the modeling of the red sequence. As mentioned at the end of Section 3, the more S0s are included in the definition of red sequence, the steeper the slope becomes, as the average color of S0s is bluer than that of ellipticals. This argues that mergers between progenitors of massive ellipticals and quiescent disk galaxies (SOs) could be quite important. In light of this uncertainty, our understanding of the merging history of massive ellipticals will benefit from better comparison of the stellar population between S0s and the outskirts of massive ellipticals. More detailed observations of on-going dry mergers at gradually higher redshift will certainly help too.

\subsection{Future Directions and Applications}

With all the above uncertainties in mind, the method proposed here and the current results can be improved in several ways. In this work, although the 2-D models in $B$ and $R$ band are tied to the ones in the $V$ band with little freedom, they are still fit separately. Simultaneous multi-band modeling should help us extract more reliable colors in more than two bands, while a forward-modeling approach using an MCMC-Bayesian method can further help understand the internal degeneracies among parameters. In addition, using a large sample of massive galaxies with well-calibrated photometry can help us more accurately constrain the $M_{*}$-size relation. It is also possible that using a single optical color is not the best way to define the average stellar population properties of different components. We might, for instance, utilize the average SED of the inner and outer components based on multi-band data (e.g., similar to La Barbera et al. 2012), but instead of using SED fitting to get the age and metallicity profiles, we can search along the red sequence for galaxies with similar SED. All these approaches will allow us to understand the offset of our results based on different colors, and significantly improve the current constraint of average merger mass ratio. A sample of $\sim 2000$ massive $\left(\log \left(M_{*} / M_{\odot}\right)>11.0\right)$ elliptical galaxies at $z<0.3$ has been selected from the Hyper-Suprime Camera (HSC) survey for a similar analysis (S. Huang et al., in preparation). As the HSC survey has much better seeing and spatial resolution, and is at least 2.5 mag deeper ( $i$ band) than CGS and SDSS, it is perfect for 2-D photometric modeling of massive galaxies. This sample also includes $\sim 80$ brightest cluster galaxies. Crucial but untouched issues, like the effect of environment or the relations of color difference with other properties, can be addressed.

Another avenue to explore is the low-level tidal features or remnants of ongoing dry mergers around nearby ellipticals, perhaps via ultra-deep imaging. Such analysis helps us understand the distribution of mass ratio of dry mergers around massive ellipticals at $z \approx 0$, and provides useful constraints on galaxy formation through comparison with simulations or semi-analytic models. In Gu et al. (2013), multi-component modeling of NGC 4889 revealed a new set of shells, which can be traced back to a recent minor dry merger. The authors estimated the mass ratio of the merger using the optical color of the shells and the tight red sequence observed in the Coma cluster. Using deeper images from the HSC survey, it is clear that recent merging events are more common around massive galaxies (Tanaka et al. , in preparation). Careful color measurements of these tidal features should provide better statistics of recent mergers than previous works. In the near future, surveys conducted with facilities such as the Large Synoptic Survey Telescope (LSST) will be even more helpful in this direction.

Next, a self-consistent model for the formation of massive galaxies must be able to correctly predict the evolution of color gradients too. Color gradients of massive galaxies at high- $z$ are becoming available (e.g., Hinkley et al. 2001; Ferreras et al. 2009; Gargiulo et al. 2011; Guo et al. 2011). If multi-band decomposition can be applied to rest-frame optical images of massive galaxies at increasingly higher redshift, we may be able to directly witness the gradual build-up of their outer envelope through minor mergers, along with the formation of their negative color gradients.

Finally, large integral-field spectroscopic surveys like MaNGA (Bundy et al. 2015) will provide direct evidence of stellar population differences between the inner and outer regions of nearby massive ellipticals. More importantly, rough star formation histories can be inferred from comparison between data and synthetic stellar population models. The photometric method proposed here can serve as an efficient and effective complement to current integral-field spectroscopic surveys.

\section{SUMMARY}

We extend the multiple-component photometric decomposition of nearby elliptical galaxies in CGS into different filters. We demonstrate that consistent three-component models can be obtained independently from images in the $B, V$, and $R$ bands, when the PSF and the sky background are properly taken into account. Using reasonable parameter constraints, we extract $B-V$ and $B-R$ colors of different photometric components. The combination of these components with slightly different color can perfectly recover the negative color gradient of massive elliptical galaxies, as well as their radial profiles of surface brightness and geometric parameters. In the recent work by Huang et al. (2013b), these photometric components relate to physical structures that were formed through different mechanisms and at different phases of the evolution of massive galaxies. To be more specific, the inner structure is similar to the massive, compact galaxies seen at $z \geq 1.5$, which most likely formed through a highly dissipative process. On the other hand, the outer component can be best explained by the gradual accretion of stellar material through many, mostly dry, mergers. In light of this evidence, we measured the average color difference between the inner and outer components for massive ellipticals in our sample $\left(M_{*} \geq 1.5 \times 10^{11} M_{\odot} ;\langle\Delta(B-V)\rangle \approx 0.10 \mathrm{mag}\right.$; $\langle\Delta(B-R)\rangle \approx 0.14 \mathrm{mag})$. By adopting a few assumptions from the two-phase formation scenario, and by deriving the $M_{*}$-color 
relations for nearby ETGs, we translated these color differences into an average merger mass ratio for the build-up of the outer envelope. According to our estimates, the average merger ratio lies between 1:5 to 1:10. This is in line with the prediction that minor, dry mergers should dominate the growth of massive elliptical galaxies during the last few Gyr. The method we proposed here only relies on decent photometric data in more than one filter, hence has great potential to be applied to existing (e.g., SDSS, CANDELS), ongoing (e.g., HSC Survey), and future (e.g., LSST) large-scale photometric surveys.

We thank Andrew Cooper for generously providing data from his work to us for comparison. This work was supported by Carnegie Observatories, Peking University, the Kavli Foundation, the Chinese Academy of Science through grant No. XDB09030102 (Emergence of Cosmological Structures) from the Strategic Priority Research Program, the National Natural Science Foundation of China through grant No. 11473002 (LCH) and No. 11403072 (ZYL), the UC Irvine School of Physical Sciences (AJB), and the World Premier International Research Center Initiative, MEXT, Japan (SH). SH thanks Prof. Q.-S. Gu and the School of Space Science and Astronomy in
Nanjing University for providing long-term support. ZYL is grateful for the support from the Shanghai Yangfan Research Grant (No. 14YF1407700).

Funding for SDSS-III has been provided by the Alfred P. Sloan Foundation, the Participating Institutions, the National Science Foundation, and the U.S. Department of Energy. The SDSS-III web site is http://www.sdss3.org. SDSS-III is managed by the Astrophysical Research Consortium for the Participating Institutions of the SDSS-III Collaboration including the University of Arizona, the Brazilian Participation Group, Brookhaven National Laboratory, University of Cambridge, University of Florida, the French Participation Group, the German Participation Group, the Instituto de Astrofisica de Canarias, the Michigan State/Notre Dame/JINA Participation Group, Johns Hopkins University, Lawrence Berkeley National Laboratory, Max Planck Institute for Astrophysics, New Mexico State University, New York University, Ohio State University, Pennsylvania State University, University of Portsmouth, Princeton University, the Spanish Participation Group, University of Tokyo, University of Utah, Vanderbilt University, University of Virginia, University of Washington, and Yale University.

\section{REFERENCES}

Arnold, J. A., Romanowsky, A. J., Brodie, J. P., et al. 2011, ApJ, 736, L26

Balcells, M., \& Peletier, R. F. 1994, AJ, 107, 135

Bédorf, J., \& Portegies Zwart, S. 2013, MNRAS, 431, 767

Bell, E. F., McIntosh, D. H., Katz, N., \& Weinberg, M. D. 2003, ApJS, 149, 289

Bell, E. F., \& de Jong, R. S. 2001, ApJ, 550, 212

Bell, E. F., Wolf, C., Meisenheimer, K., et al. 2004, ApJ, 608, 752

Bennert, V. N., Auger, M. W., Treu, T., Woo, J.-H., \& Malkan, M. A. 2011, ApJ, 742, 107

Bernardi, M., Nichol, R. C., Sheth, R. K., Miller, C. J., \& Brinkmann, J. 2006, AJ, 131, 1288

Bernardi, M., Roche, N., Shankar, F., \& Sheth, R. K. 2011, MNRAS, 412, 684

Bernardi, M., Sheth, R. K., Annis, J., et al. 2003, AJ, 125, 1882

Bernardi, M., Sheth, R. K., Nichol, R. C., Schneider, D. P., \& Brinkmann, J. 2005, AJ, 129, 61

Bluck, A. F. L., Conselice, C. J., Buitrago, F., et al. 2012, ApJ, 747, 34

Bower, R. G., Lucey, J. R., \& Ellis, R. S. 1992, MNRAS, 254, 601

Boylan-Kolchin, M., Springel, V., White, S. D. M., Jenkins, A., \& Lemson, G. 2009, MNRAS, 398, 1150

Bundy, K., Bershady, M. A., Law, D. R., et al. 2015, ApJ, 798, 7

Cappellari, M., Scott, N., Alatalo, K., et al. 2013, MNRAS, 432, 1709

Carlberg, R. G. 1984, ApJ, 286, 416

Cassata, P., Guzzo, L., Franceschini, A., et al. 2007, ApJS, 172, 270

Cerulo, P., Couch, W. J., Lidman, C., et al. 2016, MNRAS, in press (arXiv:1601.07578)

Chabrier, G. 2003, ApJ, 586, L133

Choi, J., Conroy, C., Moustakas, J., et al. 2014, ApJ, 792, 95

Coccato, L., Arnaboldi, M., \& Gerhard, O. 2013, MNRAS, 436, 1322

Coccato, L., Gerhard, O., \& Arnaboldi, M. 2010, MNRAS, 407, L26

Cooper, A. P., D’Souza, R., Kauffmann, G., et al. 2013, MNRAS, 434, 3348

Davies, R. L., Sadler, E. M., \& Peletier, R. F. 1993, MNRAS, 262, 650

de Vaucouleurs, G. 1961, ApJS, 5, 233

D’Souza, R., Kauffman, G., Wang, J., \& Vegetti, S. 2014, MNRAS, 443, 1433

Eggen, O. J., Lynden-Bell, D., \& Sandage, A. R. 1962, ApJ, 136, 748

Faber, S. M. 1973, ApJ, 179, 731

Faber, S. M., Willmer, C. N. A., Wolf, C., et al. 2007, ApJ, 665, 265

Ferreras, I., Lisker, T., Pasquali, A., \& Kaviraj, S. 2009, MNRAS, 395, 554

Ferreras, I., Trujillo, I., Mármol-Queraltó, E., et al. 2014, MNRAS, 444, 906

Franx, M., \& Illingworth, G. 1990, ApJ, 359, L41

Fritz, A., Scodeggio, M., Ilbert, O., et al. 2014, A\&A, 563, AA92

Gabor, J. M., \& Davé, R. 2012, MNRAS, 427, 1816

Gallazzi, A., Charlot, S., Brinchmann, J., White, S. D. M., \& Tremonti, C. A. 2005, MNRAS, 362, 41

Gargiulo, A., Saracco, P., \& Longhetti, M. 2011, MNRAS, 412, 1804

Gladders, M. D., López-Cruz, O., Yee, H. K. C., \& Kodama, T. 1998, ApJ, 501, 571

González Delgado, R. M., Cid Fernandes, R., García-Benito, R., et al. 2014, ApJ, 791, L16

Graves, G. J., Faber, S. M., \& Schiavon, R. P. 2009, ApJ, 693, 486

Greene, J. E., Janish, R., Ma, C.-P., et al. 2015, ApJ, 807, 11
Greene, J. E., Murphy, J. D., Comerford, J. M., Gebhardt, K., \& Adams, J. J. 2012, ApJ, 750, 32

Greene, J. E., Murphy, J. D., Graves, G. J., et al. 2013, ApJ, 776, 64

Guo, Q., White, S., Boylan-Kolchin, M., et al. 2011, MNRAS, 413, 101

Guo, Y., Giavalisco, M., Cassata, P., et al. 2011, ApJ, 735, 18

Hansen, S. M., Sheldon, E. S., Wechsler, R. H., \& Koester, B. P. 2009, ApJ, 699,1333

Häußler, B., Bamford, S. P., Vika, M., et al. 2013, MNRAS, 430, 330

Hilz, M., Naab, T., \& Ostriker, J. P. 2013, MNRAS, 429, 2924

Hilz, M., Naab, T., Ostriker, J. P., et al. 2012, MNRAS, 425, 3119

Hinkley, S., \& Im, M. 2001, ApJ, 560, L41

Hirschmann, M., Naab, T., Ostriker, J. P., et al. 2015, MNRAS, 449, 528

Ho, L. C., Li, Z.-Y., Barth, A. J., Seigar, M. S., \& Peng, C. Y. 2011, ApJS, 197, 21 (Paper I)

Huang, S., Ho, L. C., Peng, C. Y., Li, Z.-Y., \& Barth, A. J. 2013a, ApJ, 766, 47 (Paper III)

Huang, S., Ho, L. C., Peng, C. Y., Li, Z.-Y., \& Barth, A. J. 2013b, ApJ, 768, L28

Huertas-Company, M., Aguerri, J. A. L., Bernardi, M., Mei, S., \& Sánchez Almeida, J. 2011, A\&A, 525, AA157

Kaviraj, S., Huertas-Company, M., Cohen, S., et al. 2014, MNRAS, 443, 1861

Kawata, D., \& Gibson, B. K. 2003, MNRAS, 340, 908

Kelly, B. C. 2007 , ApJ, 665, 1489

Kim, D., \& Im, M. 2013, ApJ, 766, 109

Kobayashi, C. 2004, MNRAS, 347, 740

Kodama, T., Arimoto, N., Barger, A. J., \& Arag'on-Salamanca, A. 1998, A\&A, 334,99

Kormendy, J., \& Djorgovski, S. 1989, ARA\&A, 27, 235

Kormendy, J., Fisher, D. B., Cornell, M. E., \& Bender, R. 2009, ApJS, 182, 216

Kriek, M., van der Wel, A., van Dokkum, P. G., Franx, M., \& Illingworth, G. D. 2008, ApJ, 682, 896

Kuntschner, H., Emsellem, E., Bacon, R., et al. 2010, MNRAS, 408, 97

La Barbera, F., De Carvalho, R. R., De La Rosa, I. G., et al. 2010, AJ, 140, 1528

La Barbera, F., Ferreras, I., de Carvalho, R. R., et al. 2012, MNRAS, 426, 2300

Lackner, C. N., Cen, R., Ostriker, J. P., \& Joung, M. R. 2012, MNRAS, 425, 641

Lackner, C. N., \& Gunn, J. E. 2012, MNRAS, 421, 2277

Larson, R. B. 1975, MNRAS, 173, 671

Lauer, T. R., Faber, S. M., Gebhardt, K., et al. 2005, AJ, 129, 2138

Lee, J. H., Kim, M., Ree, C. H., et al. 2013, ApJ, 762, L4

Li, Z.-Y., Ho, L. C., Barth, A. J., \& Peng, C. Y. 2011, ApJS, 197, 22 (Paper II)

López-Sanjuan, C., Le Fèvre, O., Ilbert, O., et al. 2012, A\&A, 548, AA7

Lupton, R. H., Jurić, M., Ivezić, Z., et al. 2005, B.A.A.S., 37, \#133.08

MacArthur, L. A., Courteau, S., Bell, E., \& Holtzman, J. A. 2004, ApJS, 152, 175

Mei, S., Holden, B. P., Blakeslee, J. P., et al. 2009, ApJ, 690, 42

Mendel, J. T., Simard, L., Palmer, M., Ellison, S. L., \& Patton, D. R. 2014, ApJS, 210, 3

Michard, R. 2002, A\&A, 384, 763

Michard, R. 2005, A\&A, 441, 451 
Montes, M., Trujillo, I., Prieto, M. A., \& Acosta-Pulido, J. A. 2014, MNRAS, 439, 990

Newman, A. B., Ellis, R. S., Bundy, K., \& Treu, T. 2012, ApJ, 746, 162

Nicol, M.-H., Meisenheimer, K., Wolf, C., \& Tapken, C. 2011, ApJ, 727, 51

Ogando, R. L. C., Maia, M. A. G., Chiappini, C., et al. 2005, ApJ, 632, L61

Oogi, T., \& Habe, A. 2013, MNRAS, 428, 641

Oser, L., Naab, T., Ostriker, J. P., \& Johansson, P. H. 2012, ApJ, 744, 63

Oser, L., Ostriker, J. P., Naab, T., Johansson, P. H., \& Burkert, A. 2010, ApJ, 725,2312

Ownsworth, J. R., Conselice, C. J., Mortlock, A., et al. 2014, MNRAS, 445, 2198

Peletier, R. F., Davies, R. L., Illingworth, G. D., Davis, L. E., \& Cawson, M. 1990, AJ, 100, 1091

Peng, C. Y., Ho, L. C., Impey, C. D., \& Rix, H.-W. 2002, AJ, 124, 266

Peng, C. Y., Ho, L. C., Impey, C. D., \& Rix, H.-W. 2010, AJ, 139, 2097

Pipino, A., D'Ercole, A., Chiappini, C., \& Matteucci, F. 2010, MNRAS, 407, 1347

Rawle, T. D., Smith, R. J., \& Lucey, J. R. 2010, MNRAS, 401, 852

Rodriguez-Gomez, V., Pillepich, A., Sales, L. V., et al. 2016, MNRAS, in press (arXiv:1511.08804)

Ruiz, P., Trujillo, 1., \& Mármol-Queraltó, E. 2014, MNRAS, 442, 347

Ruiz, P., Trujillo, I., \& Mármol-Queraltó, E. 2015, MNRAS, 454, 1605

Rupke, D. S. N., Kewley, L. J., \& Barnes, J. E. 2010, ApJ, 710, L156
Sánchez-Blázquez, P., Gorgas, J., \& Cardiel, N. 2006, A\&A, 457, 823

Schawinski, K., Urry, C. M., Simmons, B. D., et al. 2014, MNRAS, 440, 889

Simard, L., Mendel, J. T., Patton, D. R., Ellison, S. L., \& McConnachie, A. W. 2011, ApJS, 196, 11

Skelton, R. E., Bell, E. F., \& Somerville, R. S. 2009, ApJ, 699, L9

Sonnenfeld, A., Nipoti, C., \& Treu, T. 2014, ApJ, 786, 89

Spolaor, M., Kobayashi, C., Forbes, D. A., Couch, W. J., \& Hau, G. K. T. 2010, MNRAS, 408, 272

Tal, T., van Dokkum, P. G., Nelan, J., \& Bezanson, R. 2009, AJ, 138, 1417

Tal, T., \& van Dokkum, P. G. 2011, ApJ, 731, 89

Tanaka, M., Kodama, T., Arimoto, N., et al. 2005, MNRAS, 362, 268

Taylor, E. N., Hopkins, A. M., Baldry, I. K., et al. 2015, MNRAS, 446, 2144

Vader, J. P., Vigroux, L., Lachieze-Rey, M., \& Souviron, J. 1988, A\&A, 203, 217

Vika, M., Bamford, S. P., Häußler, B., \& Rojas, A. L. 2014, MNRAS, 444, 3603

Vika, M., Bamford, S. P., Häußler, B., et al. 2013, MNRAS, 435, 623

Wang, W., Sales, L. V., Henriques, B. M. B., \& White, S. D. M. 2014, MNRAS, 442,1363

Whitaker, K. E., van Dokkum, P. G., Brammer, G., et al. 2010, ApJ, 719, 1715

Wise, M. W., \& Silva, D. R. 1996, ApJ, 461, 155

Wu, H., Shao, Z., Mo, H. J., Xia, X., \& Deng, Z. 2005, ApJ, 622, 244

Yoon, I., Weinberg, M. D., \& Katz, N. 2011, MNRAS, 414, 1625

\section{APPENDIX}

\section{A. COMPARISON OF COLOR DIFFERENCES USING 1-D COLOR PROFILES}

As mentioned in the main text, we suggest that the 2-D multi-component modeling method can help us gain more insight into the assembly history of massive galaxies through their 2-D color distribution. In our method, while the detailed surface brightness distribution and 2-D geometric information are accurately recovered, the negative color gradient can also be well described by the superposition of two distinct components that have slightly different color. As shown in Figure 2 (also see Figure 10 of RodriguezGomez et al. 2016), the accreted component (represented by our "outer" component) and the in-situ component ("inner" component) overlap substantially in radius: the accreted component starts to dominate over the in-situ component from $2 R_{\mathrm{e}}$ to $4 R_{\mathrm{e}}$, while still contributing a significant fraction of stellar mass inside $R_{\mathrm{e}}$. Hence, it is intrinsically difficult to extract the average color of both the in-situ and accreted components using just the 1-D color profiles. However, as the 1-D color gradient is still commonly used, here we briefly compare the color difference between sub-components as derived from our 2-D method and the one derived from 1-D color profiles. As shown in Figure 5, due to the strong impact from uncertainty of background subtraction, the color profile in low surface brightness regions typically has large uncertainties. Since we do need a large dynamical range in radius to estimate a meaningful color difference to compare with our results, we decide to use the 1-D color profiles based on the best, multi-component models instead of the observed one.

The PSF convolution is removed from the model to help us recover the intrinsic color at the center (although the radius range we use is not affected by the PSF). And the same set of isophotes used to extract the 1-D surface brightness profile from data is applied to the model images in both bands (This is done using the "forced photometry" mode of the ELLIPSE software.) Compared to the color profiles derived from the original images, the ones from the best models do not show a sudden change at large radii. For comparison, we use two methods to define the range of radius: (1) we use the color difference between 3 and 10 (20, 30) Kpc; (2) we estimate the color difference between 0.5 and $2(3,4)$ times $R_{\mathrm{e}}$ (from single Sérsic model). The results are summarized in the left and middle panel of Figure A1. In general, there is a scattered correlation between these two methods, and the trends become clearer for larger dynamical range in radius. But, as expected, the typical color difference from 1-D profile across all the radius range is smaller than the one between two sub-components. For $B-V$ color, the median color difference between 3 and 10 (20, 30) Kpc is 0.04 ( 0.06 , $0.07) \mathrm{mag}$; the corresponding values for $B-R$ color are $0.04(0.07,0.08)$ mag. When relative radius is used, the color difference between $0.5 R_{\mathrm{e}}$ and $2(3,4) R_{\mathrm{e}}$ is $0.04(0.07,0.07) \mathrm{mag}$ for $B-V$, and $0.05(0.07,0.08) \mathrm{mag}$ for $B-R$. D'Souza et al. (2014) provided median $g-r$ color profiles of massive, early-type (high concentration index) galaxies using stacked SDSS images. The $g-r$ color can be approximately converted to $B-V$ color using empirical relations by Lupton et al. (2005). Assuming $\Delta(B-V)=0.891 \times \Delta(g-r)$, the expected $B-V$ color difference between 3 and $10(20,30) \mathrm{Kpc}$ is $0.05(0.07,0.08)$ mag. Meanwhile, La Barbera et al. (2012) provided median $g-r$ color profiles for massive galaxies scaled relative to $R_{\mathrm{e}}$. Using the same assumption, the expected $B-V$ color difference between $0.5 R_{\mathrm{e}}$ and $2(3,4) R_{\mathrm{e}}$ is $0.04(0.06,0.07) \mathrm{mag}$. Although these profiles were derived using different methods, they are quite consistent with our results. This suggests that our multi-component models reliably capture the color profile in this radius range. In the right panel of Figure $A 1$, we also show the $M_{*}-\Delta(B-V)$ and $M_{*}-\Delta(B-R)$ relations in form similar to Figure 10 . 

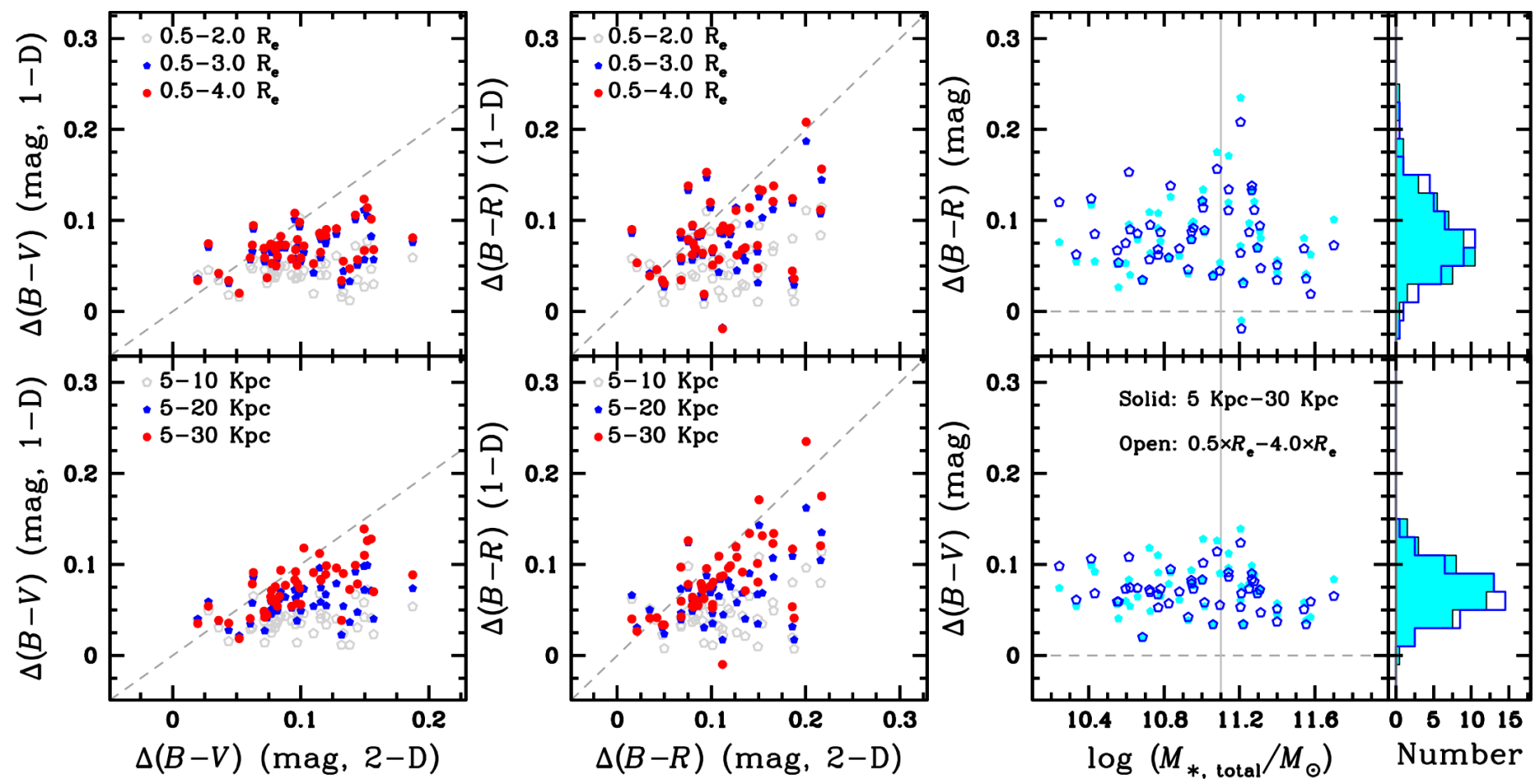

FIG. A1.- The left $(B-V)$ and middle $(B-R)$ panels compare the color difference between the inner and outer components with the color differences derived from 1-D color profiles based on the best 2-D models. On the lower panels, the color difference is defined by fixed absolute radius, while the upper ones use distance defined relative to effective radius. A grey dashed line shows the one-to-one relation. The right panel shows the relation between stellar mass and color difference derived from 1-D color profiles. 
TABLE 1

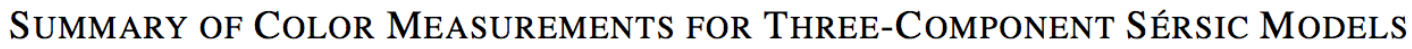

\begin{tabular}{|c|c|c|c|c|c|c|c|c|c|}
\hline \multirow{2}{*}{$\begin{array}{c}\text { Galaxy } \\
\text { (1) }\end{array}$} & \multicolumn{3}{|c|}{$\log \left(M_{*} / M_{\odot}\right)$} & \multirow{2}{*}{$\begin{array}{l}\text { Color Type } \\
\text { (5) }\end{array}$} & \multicolumn{4}{|c|}{ Color of Each Component (mag) } & \multirow{2}{*}{$\begin{array}{l}\Delta\left(\text { Color }_{\text {inner-outer }}\right. \\
\text { (mag) } \\
(10)\end{array}$} \\
\hline & $\begin{array}{l}\text { Total } \\
\text { (2) }\end{array}$ & Inner & $\begin{array}{l}\text { Outer } \\
(4)\end{array}$ & & $\begin{array}{l}\text { Center } \\
\text { (6) }\end{array}$ & $\begin{array}{l}\text { Intermediate } \\
\text { (7) }\end{array}$ & $\begin{array}{l}\text { Outer } \\
(8)\end{array}$ & $\begin{array}{l}\text { Inner } \\
(9)\end{array}$ & \\
\hline \multirow[t]{4}{*}{ ESO185-G054 } & $11.65 \pm 0.08$ & 10.87 & 11.56 & $B-V$ fixed & 0.97 & 1.04 & 0.89 & 1.00 & 0.11 \\
\hline & & & & $B-V$ free & 0.97 & 1.02 & 0.90 & 0.99 & 0.10 \\
\hline & & & & $B-R$ fixed & 1.65 & 1.72 & 1.58 & 1.68 & 0.10 \\
\hline & & & & $B-R$ free & 1.65 & 1.72 & 1.58 & 1.68 & 0.10 \\
\hline \multirow[t]{4}{*}{ IC 1459} & $11.32 \pm 0.22$ & 11.17 & 10.80 & $B-V$ fixed & 0.94 & 0.90 & 0.72 & 0.91 & 0.19 \\
\hline & & & & $B-V$ free & 0.94 & 0.90 & 0.72 & 0.91 & 0.19 \\
\hline & & & & $B-R$ fixed & 1.55 & 1.46 & 1.26 & 1.48 & 0.22 \\
\hline & & & & $B-R$ free & 1.55 & 1.46 & 1.26 & 1.48 & 0.22 \\
\hline \multirow[t]{4}{*}{ IC 2597} & $10.95 \pm 0.22$ & 10.35 & 10.82 & $B-V$ fixed & 0.97 & 1.22 & 0.83 & 1.01 & 0.18 \\
\hline & & & & $B-V$ free & 0.97 & 1.12 & 0.84 & 0.99 & 0.16 \\
\hline & & & & $B-R$ fixed & 1.63 & 1.89 & 1.49 & 1.67 & 0.18 \\
\hline & & & & $B-R$ free & 1.63 & 1.83 & 1.50 & 1.66 & 0.17 \\
\hline \multirow[t]{4}{*}{ IC 3370} & $10.97 \pm 0.06$ & 10.63 & 10.71 & $B-V$ fixed & 0.99 & 1.12 & 0.81 & 1.06 & 0.25 \\
\hline & & & & $B-V$ free & 0.97 & 1.13 & 0.82 & 1.05 & 0.23 \\
\hline & & & & $B-R$ fixed & 1.63 & 2.02 & 1.46 & 1.84 & 0.38 \\
\hline & & & & $B-R$ free & 1.62 & 2.03 & 1.46 & 1.84 & 0.38 \\
\hline \multirow[t]{4}{*}{ IC 3896} & $11.16 \pm 0.08$ & 10.84 & 10.86 & $B-V$ fixed & 1.10 & 1.03 & 0.93 & 1.05 & 0.11 \\
\hline & & & & $B-V$ free & 1.11 & 1.01 & 0.95 & 1.04 & 0.09 \\
\hline & & & & $B-R$ fixed & 1.91 & 1.81 & 1.72 & 1.84 & 0.12 \\
\hline & & & & $B-R$ free & 1.91 & 1.81 & 1.72 & 1.84 & 0.12 \\
\hline \multirow[t]{4}{*}{ IC 4296} & $11.70 \pm 0.22$ & 11.45 & 11.35 & $B-V$ fixed & 1.02 & 0.94 & 0.86 & 0.95 & 0.09 \\
\hline & & & & $B-V$ free & 1.01 & 0.95 & 0.84 & 0.96 & 0.12 \\
\hline & & & & $B-R$ fixed & 1.67 & 1.61 & 1.45 & 1.62 & 0.17 \\
\hline & & & & $B-R$ free & 1.67 & 1.61 & 1.47 & 1.62 & 0.15 \\
\hline \multirow[t]{4}{*}{ IC 4329} & $11.68 \pm 0.22$ & 10.81 & 11.60 & $B-V$ fixed & 0.88 & 0.98 & 0.81 & 0.91 & 0.11 \\
\hline & & & & $B-V$ free & 0.88 & 0.91 & 0.82 & 0.89 & 0.07 \\
\hline & & & & $B-R$ fixed & 1.50 & 1.64 & 1.41 & 1.54 & 0.13 \\
\hline & & & & $B-R$ free & 1.50 & 1.57 & 1.43 & 1.52 & 0.09 \\
\hline \multirow[t]{4}{*}{ IC 4797} & $10.81 \pm 0.22$ & 10.45 & 10.56 & $B-V$ fixed & 0.90 & 0.85 & 0.78 & 0.88 & 0.10 \\
\hline & & & & $B-V$ free & 0.90 & 0.85 & 0.78 & 0.88 & 0.10 \\
\hline & & & & $B-R$ fixed & 1.54 & 1.57 & 1.44 & 1.55 & 0.11 \\
\hline & & & & $B-R$ free & 1.54 & 1.57 & 1.44 & 1.55 & 0.11 \\
\hline
\end{tabular}

FIG. TABLE 1.- Summary of stellar mass estimations, the color of each component, and the color difference between the inner and outer components for Es included in this work. For each galaxy, the $B-V$ and $B-R$ color information with fixed or free sky background are shown in different rows. The free-background ones are recommended. Col. (1) Galaxy name. Col. (2) Total stellar mass of the galaxy. Col. (3) Stellar mass of the inner component. Col. (4) Stellar mass of the outer component. Col. (5) Type of color. Col. (6) Color of the center component. Col. (7) Color of the intermediate component. Col. (8) Color of the outer component. Col. (9) Color of the inner component. Col. (10) The color difference between the inner and outer components. The full catalog can be downloaded from https://github.com/dr-guangtou/cgs_colorgrad/raw/master/table1.pdf 